\title{
Perfect Equilibria in Games of Incomplete Information
}

\author{
Oriol Carbonell-Nicolau*
}

February 2017

\begin{abstract}
We obtain conditions on the primitives of a Bayesian game with infinitely many types and/or strategies that ensure the existence of a perfect Bayes-Nash equilibrium. The main existence results are illustrated in the context of all-pay auctions.
\end{abstract}

Keywords: infinite game of incomplete information, perfect Bayes-Nash equilibrium, payoff security.

\section{Introduction}

The notion of perfect equilibrium was introduced by Selten [23]. In normal-form games with complete information, Selten's [23] perfection refines the Nash equilibrium concept by requiring that equilibrium strategies be immune to slight trembles in the execution of the players' actions. The standard definition of perfect equilibrium for normal-form games with finite action spaces (see, e.g., van Damme [26]) can be extended to normal-form games with infinitely many actions, and these extensions have been studied by several authors (see, e.g., Al-Najjar [2], Simon and Stinchcombe [24], Carbonell-Nicolau [8, 9, 10, 12], Carbonell-Nicolau and McLean [13, 14, 15], and Bajoori et al. [4]).

This paper considers an extension of the notion of perfection to normal-form games of incomplete information, also called Bayesian games, that refines the standard Bayes-Nash equilibrium concept. Roughly, a Bayes-Nash equilibrium is perfect if there are nearby BayesNash equilibria in slightly perturbed Bayesian games in which each type of each player makes slight mistakes in the execution of a strategy. Conditions on primitives are given under which a Bayesian game with infinitely many types and/or actions, and possibly with payoff discontinuities in type and action profiles, possesses a perfect Bayes-Nash equilibrium. The existence results are illustrated in the context of all-pay auctions.

\section{Preliminaries}

Throughout the paper, the following definitions will be adopted. If $A$ is a metric space, then $\mathscr{B}(A)$ will denote the $\sigma$-algebra of Borel subsets of $A, \Delta(A)$ will represent the set of Borel probability measures on $A$, and $C(A)$ will denote the set of all bounded continuous real-valued functions on $A$.

\footnotetext{
*Department of Economics, Rutgers University, 75 Hamilton Street, New Brunswick, NJ 08901. E-mail: carbonell-nicolau@rutgers.edu.
} 
Definition 1. The $w$-topology on $\Delta(A)$ is defined as the coarsest topology for which all the functionals in

$$
\left\{\left[\left[\mu \mapsto \int_{A} f(a) \mu(d a)\right]: \Delta(A) \rightarrow \mathbb{R}\right]: f \in C(A)\right\}
$$

are continuous.

We will refer to convergence of measures in $\Delta(A)$ with respect to the $w$-topology as weak convergence of measures and we will write $\mu^{n} \underset{w}{\rightarrow} \mu$ to indicate that the sequence of measures $\left(\mu^{n}\right)$ converges weakly to $\mu$.

If $A$ is a complete, separable metric space, the $w$-topology on $\Delta(A)$ is metrizable, and the Prokhorov metric defines a compatible metric ( $c f$. Prokhorov [21, Theorem 1.11]).

\subsection{Games and strategies}

Definition 2. A normal-form game (or simply a game) is a collection $G=\left(Z_{i}, g_{i}\right)_{i=1}^{N}$, where $N$ is a finite number of players, $Z_{i}$ is a nonempty set of actions for player $i$, and $g_{i}: Z \rightarrow \mathbb{R}$ represents player $i$ 's payoff function, defined on the set of action profiles $Z:=\times_{i=1}^{N} Z_{i}$. The game $G$ is called a metric game (resp. a compact game) if each $Z_{i}$ is a metric (resp. compact) space. A compact metric game $G=\left(Z_{i}, g_{i}\right)_{i=1}^{N}$ is called a Borel game if each $g_{i}$ is bounded and $(\mathscr{B}(Z), \mathscr{B}(\mathbb{R}))$-measurable.

Throughout the sequel, given $N$ sets $Z_{1}, \ldots, Z_{N}$, we adhere to the following conventions, which are standard in the literature, even though they sometimes entail abuses of notation: for $i \in\{1, \ldots, N\}, Z_{-i}:=\times_{j \neq i} Z_{j}$; given $i$, the set $\times_{j=1}^{N} Z_{j}$ is sometimes represented as $Z_{i} \times Z_{-i}$, and we sometimes write $z=\left(z_{i}, z_{-i}\right) \in Z_{i} \times Z_{-i}$ for a member $z$ of $\times_{j=1}^{N} Z_{j}$.

Definition 3. A Bayesian game is a collection

$$
\Gamma=\left(T_{i}, X_{i}, u_{i}, p\right)_{i=1}^{N},
$$

where

- $\{1, \ldots, N\}$ is a finite set of players;

- $T_{i}$ is a nonempty, compact, metric space of types for player $i$;

- $X_{i}$ is a nonempty, compact, metric space of actions for player $i$;

- $u_{i}$ is a real-valued map on $T \times X$, where $T:=\times_{i=1}^{N} T_{i}$ and $X:=\times_{i=1}^{N} X_{i}$; it represents player $i$ 's payoff function, and it is assumed bounded and $(\mathscr{B}(T \times X), \mathscr{B}(\mathbb{R}))$-measurable; and

- $p$ is a probability measure on $(T, \mathscr{B}(T))$ describing the players' common priors over type profiles.

For each $i \in\{1, \ldots, N\}$, let $p_{i}$ be the marginal probability measure induced by $p$ on $T_{i}$, i.e., the probability measure in $\Delta\left(T_{i}\right)$ defined by

$$
p_{i}(S):=p\left(S \times T_{-i}\right), \quad \text { for every } S \in \mathscr{B}\left(T_{i}\right) .
$$

Definition 4. Let $\Gamma=\left(T_{i}, X_{i}, u_{i}, p\right)_{i=1}^{N}$ be a Bayesian game. A pure strategy for a player $i$ in $\Gamma$ is a $\left(\mathscr{B}\left(T_{i}\right), \mathscr{B}\left(X_{i}\right)\right)$-measurable map $s_{i}: T_{i} \rightarrow X_{i}$ with the interpretation that, upon learning her type $t_{i} \in T_{i}$, a player $i$ selects the action $s_{i}\left(t_{i}\right)$ from the set $X_{i}$.

Let $\mathscr{P}_{i}$ denote the set of pure strategies for player $i$, and set $\mathscr{P}:=\times_{i=1}^{N} \mathscr{P}_{i}$. 
Definition 5. Let $\Gamma=\left(T_{i}, X_{i}, u_{i}, p\right)_{i=1}^{N}$ be a Bayesian game. A distributional strategy for player $i$ in $\Gamma$ is a probability measure $\sigma_{i}$ in $\Delta\left(T_{i} \times X_{i}\right)$ such that

$$
\sigma_{i}\left(A \times X_{i}\right)=p_{i}(A), \quad \text { for all } A \in \mathscr{B}\left(T_{i}\right) .
$$

Let $\mathscr{D}_{i}$ represent the set of distributional strategies for player $i$, and define $\mathscr{D}:=\times_{i=1}^{N} \mathscr{D}_{i}$.

Given $\sigma_{i} \in \mathscr{D}_{i}$, the map $t_{i} \in T_{i} \mapsto \sigma_{i}\left(\cdot \mid t_{i}\right) \in \Delta\left(X_{i}\right)$ will denote a corresponding version of the regular conditional probability measure on $X_{i}$.

Definition 6. A distributional strategy $\sigma_{i} \in \mathscr{D}_{i}$ is strictly positive if for each $t_{i} \in T_{i}, \sigma_{i}\left(V \mid t_{i}\right)>$ 0 for every nonempty open set $V$ in $X_{i}$.

The set of all strictly positive distributional strategies in $\mathscr{D}_{i}$ is denoted by $\widehat{\mathscr{D}}_{i}$, and the Cartesian product $\times_{j=1}^{N} \widehat{\mathscr{D}}_{j}$ is denoted by $\widehat{\mathscr{D}}$. Each $\mathscr{D}_{i}$ will be endowed with the relative $w$-topology, and $\mathscr{D}$ will be endowed with the corresponding product topology.

Given a Bayesian game $\Gamma=\left(T_{i}, X_{i}, u_{i}, p\right)_{i=1}^{N}$, define the normal-form game

$$
G_{\Gamma}:=\left(\mathscr{D}_{i}, U_{i}\right)_{i=1}^{N},
$$

where $U_{i}: \mathscr{D} \rightarrow \mathbb{R}$ is given by

$$
U_{i}\left(\sigma_{1}, \ldots, \sigma_{N}\right):=\int_{T} \int_{X_{N}} \cdots \int_{X_{1}} u_{i}(t, x) \sigma_{1}\left(d x_{1} \mid t_{1}\right) \cdots \sigma_{N}\left(d x_{N} \mid t_{N}\right) p(d t) .
$$

Given $\alpha=\left(\alpha_{1}, \ldots, \alpha_{N}\right) \in[0,1)^{N}$ and $\mu=\left(\mu_{1}, \ldots, \mu_{N}\right) \in \widehat{\mathscr{D}}$, define the normal-form game

$$
G_{\Gamma}^{(\alpha, \mu)}:=\left(\mathscr{D}_{i}, U_{i}^{(\alpha, \mu)}\right)_{i=1}^{N},
$$

where $U_{i}^{(\alpha, \mu)}: \mathscr{D} \rightarrow \mathbb{R}$ is defined by

$$
U_{i}^{(\alpha, \mu)}\left(\sigma_{1}, \ldots, \sigma_{N}\right):=U_{i}\left(\left(1-\alpha_{1}\right) \sigma_{1}+\alpha_{1} \mu_{1}, \ldots,\left(1-\alpha_{N}\right) \sigma_{N}+\alpha_{N} \mu_{N}\right) .
$$

Every pure strategy in $\mathscr{P}_{i}$ induces a corresponding "pure" distributional strategy in $\mathscr{D}_{i}$ in a natural way. If $s_{i} \in \mathscr{P}_{i}$, define $\sigma_{i}^{s_{i}} \in \mathscr{D}_{i}$ as follows: for $t_{i} \in T_{i}$ and $A \in \mathscr{B}\left(X_{i}\right)$,

$$
\sigma_{i}^{s_{i}}\left(A \mid t_{i}\right):=\delta_{s_{i}\left(t_{i}\right)}(A),
$$

where $\delta_{s_{i}\left(t_{i}\right)} \in \Delta\left(X_{i}\right)$ denotes the Dirac measure concentrated on the point $s_{i}\left(t_{i}\right)$.

If $s_{i} \in \mathscr{P}_{i}$ and $\sigma_{-i} \in \mathscr{D}_{-i}$, then define

$$
U_{i}\left(s_{i}, \sigma_{-i}\right):=\int_{T} \int_{X_{N}} \cdots \int_{X_{1}} u_{i}\left(t,\left(s_{i}\left(t_{i}\right), x_{-i}\right)\right)\left[\prod_{j \neq i} \sigma_{j}\left(d x_{j} \mid t_{j}\right)\right] p(d t),
$$

and note that

$$
U_{i}\left(s_{i}, \sigma_{-i}\right)=U_{i}\left(\sigma_{i}^{s_{i}}, \sigma_{-i}\right)
$$

\subsection{Equilibrium}

Definition 7. A Bayes-Nash equilibrium of a Bayesian game $\Gamma=\left(T_{i}, X_{i}, u_{i}, p\right)_{i=1}^{N}$ is a Nash equilibrium of the game $G_{\Gamma}$ defined in (1), i.e., a profile $\left(\sigma_{1}, \ldots, \sigma_{N}\right) \in \mathscr{D}$ such that for each $i$,

$$
U_{i}\left(\sigma_{i}, \sigma_{-i}\right) \geq U_{i}\left(v_{i}, \sigma_{-i}\right), \text { for all } v_{i} \in \mathscr{D}_{i}
$$


Definition 8. A Bayes-Nash equilibrium $\sigma$ of a Bayesian game $\Gamma=\left(T_{i}, X_{i}, u_{i}, p\right)_{i=1}^{N}$ is perfect if there exist sequences $\left(\alpha^{n}\right),\left(\mu^{n}\right)$, and $\left(\sigma^{n}\right)$ such that the following holds for each $n: \alpha^{n} \in$ $(0,1)^{N}, \mu^{n} \in \widehat{\mathscr{D}}$, and $\sigma^{n}$ is a Nash equilibrium of the game $G_{\Gamma}^{\left(\alpha^{n}, \mu^{n}\right)}$ defined in (2), and in addition $\alpha^{n} \rightarrow 0$ and $\sigma^{n} \underset{w}{\rightarrow} \sigma$.

In the special case of complete information games (i.e., when type spaces are singletons), this definition collapses to the notion of perfection considered in Al-Najjar [2], CarbonellNicolau [8, 9, 10, 12], Carbonell-Nicolau and McLean [13, 14, 15], and the strong notion of perfection defined in Simon and Stinchcombe [24].

\section{Existence of perfect equilibrium}

To begin, we recall the definition of payoff security.

Definition 9 (Reny [22]). A topological game $\left(Z_{i}, g_{i}\right)_{i=1}^{N}$ is payoff secure if for each $\varepsilon>0$, $z \in \times{ }_{i=1}^{N} Z_{i}$, and $i$, there exists a $y_{i} \in Z_{i}$ and a neighborhood $V_{z_{-i}}$ of $z_{-i}$ such that $g_{i}\left(y_{i}, y_{-i}\right)>$ $g_{i}(z)-\varepsilon$ for every $y_{-i} \in V_{z_{-i}}$.

The following definition extends Condition (A) in Carbonell-Nicolau [8, 9] to games with incomplete information and strengthens the notion of uniform payoff security for incomplete information games introduced in Carbonell-Nicolau and McLean [16].

Definition 10. The Bayesian game $\left(T_{i}, X_{i}, u_{i}, p\right)_{i=1}^{N}$ satisfies strong uniform payoff security if there exists $\mu=\left(\mu_{1}, \ldots, \mu_{N}\right) \in \widehat{\mathscr{D}}$ such that for each $i$ and $\varepsilon>0$ there is a sequence $\left(f^{k}\right)$ of $\left(\mathscr{B}\left(X_{i}\right), \mathscr{B}\left(X_{i}\right)\right)$-measurable maps $f^{k}: X_{i} \rightarrow X_{i}$ satisfying the following:

(a) For each $k$ and $(t, x) \in T \times X$, there exists a neighborhood $V_{x_{-i}}$ of $x_{-i}$ such that

$$
u_{i}\left(t,\left(f^{k}\left(x_{i}\right), y_{-i}\right)\right)>u_{i}(t, x)-\varepsilon, \quad \text { for all } y_{-i} \in V_{x_{-i}} .
$$

(b) For each $\left(t, x_{-i}\right) \in T \times X_{-i}$, there is a subset $Y_{i}$ of $X_{i}$ with $\mu_{i}\left(Y_{i} \mid t_{i}\right)=1$ satisfying the following: for each $x_{i} \in Y_{i}$, there exists $K$ such that for all $k \geq K$, there is a neighborhood $V_{x_{-i}}^{\prime}$ of $x_{-i}$ such that

$$
u_{i}\left(t,\left(f^{k}\left(x_{i}\right), y_{-i}\right)\right)<u_{i}\left(t,\left(x_{i}, y_{-i}\right)\right)+\varepsilon, \quad \text { for all } y_{-i} \in V_{x_{-i}}^{\prime}
$$

The proofs of the next two lemmas are relegated to Section A.

Lemma 1. Suppose that the Bayesian game $\Gamma=\left(T_{i}, X_{i}, u_{i}, p\right)_{i=1}^{N}$ satisfies strong uniform payoff security. If $p$ is absolutely continuous with respect to $p_{1} \otimes \cdots \otimes p_{N}$, then there exists $\mu \in \widehat{D}$ such that the game $G_{\Gamma}^{(\alpha, \mu)}$ defined in (2) is payoff secure for each $\alpha \in[0,1)^{N}$.

Lemma 2. Given a Bayesian game $\left(T_{i}, X_{i}, u_{i}, p\right)_{i=1}^{N}$, suppose that for each $t \in T$, the map $\sum_{i=1}^{N} u_{i}(t, \cdot): X \rightarrow \mathbb{R}$ is upper semicontinuous. Suppose further that $p$ is absolutely continuous with respect to $p_{1} \otimes \cdots \otimes p_{N}$. Then the map $\sum_{i=1}^{N} U_{i}(\cdot): \mathscr{D} \rightarrow \mathbb{R}$ is upper semicontinuous.

Our first main existence result is Theorem 1.

Theorem 1. Suppose that the Bayesian game $\Gamma=\left(T_{i}, X_{i}, u_{i}, p\right)_{i=1}^{N}$ satisfies strong uniform payoff security and that for each $t \in T$, the map $\sum_{i=1}^{N} u_{i}(t, \cdot): X \rightarrow \mathbb{R}$ is upper semicontinuous. If $p$ is absolutely continuous with respect to $p_{1} \otimes \cdots \otimes p_{N}$, then $\Gamma$ possesses a perfect Bayes-Nash equilibrium. 
Proof. For each $n \in \mathbb{N}$, let $\alpha^{n}:=\left(\frac{1}{n}, \ldots, \frac{1}{n}\right)$. By Lemma 1 , there exists $\mu \in \widehat{\mathscr{D}}$ such that for each $n$, the game $G_{\Gamma}^{\left(\alpha^{n}, \mu\right)}$ is payoff secure. In addition, Lemma 2 implies that the map $\sum_{i=1}^{N} U_{i}(\cdot)$ : $\mathscr{D} \rightarrow \mathbb{R}$ is upper semicontinuous, implying that for each $n$ the map $\sum_{i=1}^{N} U_{i}^{\left(\alpha^{n}, \mu\right)}(\cdot): \mathscr{D} \rightarrow \mathbb{R}$ is upper semicontinuous. Consequently, since each $\mathscr{D}_{i}$ is a compact ( $c f$. Milgrom and Weber [19, p. 626]), convex subset of a topological vector space, it follows from Proposition 3.2 and Theorem 3.1 of Reny [22] that the game $G_{\Gamma}^{\left(\alpha^{n}, \mu\right)}$ has a Nash equilibrum $\sigma^{n}$ for each $n$. Now, since the sequence $\left(\sigma^{n}\right)$ lies in $\mathscr{D}$ and since $\mathscr{D}$ is sequentially compact, one may write (passing to a subsequence if necessary) $\sigma^{n} \underset{w}{\rightarrow} \sigma$ for some $\sigma \in \mathscr{D}$. It follows that $\sigma$ is a perfect Bayes-Nash equilibrium of $\Gamma$ if it is a Bayes-Nash equilibrium of $\Gamma$.

We shall assume that $\sigma$ is not a Bayes-Nash equilibrium of $\Gamma$ and derive a contradiction. Because $\sigma^{n} \underset{w}{\rightarrow} \sigma$ and since each $U_{i}$ is bounded, we have $\left(\sigma^{n},\left(U_{1}\left(\sigma^{n}\right), \ldots, U_{N}\left(\sigma^{n}\right)\right)\right) \rightarrow$ $\left(\sigma,\left(\beta_{1}, \ldots, \beta_{N}\right)\right)$ for some $\left(\beta_{1}, \ldots, \beta_{N}\right) \in \mathbb{R}^{N}$. If $\sigma$ is not a Nash equilibrium of the game $G_{\Gamma}$ defined in (1), then, since $G_{\Gamma}$ satisfies better-reply security as defined in Reny [22] (by Lemma 1, Lemma 2, and by Proposition 3.2 in Reny [22]), it follows that there exist $i, \sigma_{i}^{*} \in \mathscr{D}_{i}$, a neighborhood $V_{\sigma_{-i}}$ of $\sigma_{-i}$, and $\zeta>0$ such that

$$
U_{i}\left(\sigma_{i}^{*}, \sigma_{-i}^{\prime}\right) \geq \beta_{i}+\zeta, \quad \text { for all } \sigma_{-i}^{\prime} \in V_{\sigma_{-i}} .
$$

Therefore, since $U_{i}\left(\sigma^{n}\right) \rightarrow \beta_{i}$, there exist $\zeta^{\prime}>0$ and $\bar{n}$ such that

$$
U_{i}\left(\sigma_{i}^{*}, \sigma_{-i}^{n}\right)>U_{i}\left(\sigma^{n}\right)+\zeta^{\prime}, \quad \text { for all } n \geq \bar{n} .
$$

Consequently, there exists $n^{\prime}$ such that

$$
U_{i}\left(\left(1-\alpha_{i}^{n}\right) \sigma_{i}^{*}+\alpha_{i}^{n} \mu_{i}, \sigma_{-i}^{n}\right)>U_{i}\left(\sigma^{n}\right), \quad \text { for all } n \geq n^{\prime},
$$

contradicting that $\sigma^{n}$ is a Nash equilibrium of $G_{\Gamma}^{\left(\alpha^{n}, \mu\right)}$ for each $n$.

In the remainder of this section, we furnish a variant of Theorem 1 in which uniform payoff security (as formulated in Definition 10) is replaced by conditions that do not require an explicit construction of the $\left(\mathscr{B}\left(X_{i}\right), \mathscr{B}\left(X_{i}\right)\right)$-measurable maps $f^{k}$.

We begin with some preliminaries. Given a Bayesian game $\left(T_{i}, X_{i}, u_{i}, p\right)_{i=1}^{N}$, let $A_{i}$ be the set of all accumulation points of $X_{i}$ (i.e., the set of all points $x_{i} \in X_{i}$ such that $\left(V_{x_{i}} \backslash\left\{x_{i}\right\}\right) \cap X_{i} \neq \varnothing$ for every neighborhood $V_{x_{i}}$ of $x_{i}$ ). Since $X_{i}$ is compact and metric, it can be written as a disjoint union $A_{i} \cup K_{i}$, where $A_{i}$ is closed and dense in itself (i.e., with no isolated points) and $K_{i}$ is countable (see, e.g., Aliprantis and Border [1, Lemma 12.19]).

Definition 11. Suppose that $\Gamma=\left(T_{i}, X_{i}, u_{i}, p\right)_{i=1}^{N}$ is a Bayesian game and let $Y_{1} \subseteq X_{1}, \ldots, Y_{N} \subseteq$ $X_{N}$. The game $\Gamma$ is said to satisfy entire payoff security over $\times_{i=1}^{N} Y_{i}$ if for each $i, \varepsilon>0$, and $x_{i} \in Y_{i}$, and for every neighborhood $V_{x_{i}}$ of $x_{i}$, there exist $y_{i} \in V_{x_{i}}$ and a neighborhood $V_{x_{i}}^{\prime}$ of $x_{i}$ such that for every $\left(t, z_{-i}\right) \in T \times X_{-i}$, there is a neighborhood $V_{z_{-i}}$ of $z_{-i}$ such that

$$
u_{i}\left(t,\left(y_{i}, y_{-i}\right)\right)>u_{i}\left(t,\left(z_{i}^{\prime}, z_{-i}\right)\right)-\varepsilon, \quad \text { for all }\left(z_{i}^{\prime}, y_{-i}\right) \in V_{x_{i}}^{\prime} \times V_{z_{-i}} .
$$

Definition 12. Suppose that $\Gamma=\left(T_{i}, X_{i}, u_{i}, p\right)_{i=1}^{N}$ is a Bayesian game and let $Y_{1} \subseteq X_{1}, \ldots, Y_{N} \subseteq$ $X_{N}$. The game $\Gamma$ is said to satisfy generic entire payoff security over $\times{ }_{i=1}^{N} Y_{i}$ if there exist $Z_{1} \subseteq Y_{1}, \ldots, Z_{N} \subseteq Y_{N}$ such that $Y_{i} \backslash Z_{i}$ is countable for each $i, \Gamma$ is entirely payoff secure over $\times_{i=1}^{N} Z_{i}$, and the following condition is satisfied: for each $i, \varepsilon>0$, and $x_{i} \in Y_{i} \backslash Z_{i}$, there exists $y_{i} \in X_{i}$ such that for every $\left(t, z_{-i}\right) \in T \times X_{-i}$, there is a neighborhood $V_{z_{-i}}$ of $z_{-i}$ such that

$$
u_{i}\left(t,\left(y_{i}, y_{-i}\right)\right)>u_{i}\left(t,\left(x_{i}, z_{-i}\right)\right)-\varepsilon, \text { for all } y_{-i} \in V_{z_{-i}} .
$$


Definition 13. A Bayesian game $\Gamma=\left(T_{i}, X_{i}, u_{i}, p\right)_{i=1}^{N}$ is said to satisfy generic entire payoff security if it is entirely payoff secure over $\times_{i=1}^{N} K_{i}$ and generically entirely payoff secure over $\times{ }_{i=1}^{N} A_{i}$.

Recall that the set of all strictly positive distributional strategies in $\mathscr{D}_{i}$ (cf. Definition 6) is denoted by $\widehat{\mathscr{D}}_{i}$. Let $\widetilde{\mathscr{D}}_{i}$ be the set of members $\sigma_{i}$ of $\mathscr{D}_{i}$ such that for each $t_{i} \in T_{i}, \sigma_{i}\left(\left\{x_{i}\right\} \mid t_{i}\right)=0$ and $\sigma_{i}\left(N_{\epsilon}\left(x_{i}\right) \mid t_{i}\right)>0$ for every $x_{i} \in A_{i}$ and $\epsilon>0$ (where $N_{\epsilon}\left(x_{i}\right)$ denotes the $\epsilon$-neighborhood of $\left.x_{i}\right)$, and $\sigma_{i}\left(\left\{x_{i}\right\} \mid t_{i}\right)>0$ for every $x_{i} \in K_{i}$. Observe that $\widetilde{\mathscr{D}}_{i} \subseteq \widehat{\mathscr{D}}_{i}$. In addition, $\widetilde{\mathscr{D}}_{i}$ is nonempty (see, e.g., Parthasarathy et al. [20, Corollary 6.2]). Define $\widetilde{\mathscr{D}}_{i=} \times_{i=1}^{N} \widetilde{\mathscr{D}}_{i}$.

Definition 14. The Bayesian game $\left(T_{i}, X_{i}, u_{i}, p\right)_{i=1}^{N}$ satisfies generic local equi-upper semicontinuity if there exists $\mu=\left(\mu_{1}, \ldots, \mu_{N}\right) \in \widetilde{\mathscr{D}}$ such that for each $i$ and $\left(t, x_{-i}\right) \in T \times X_{-i}$, there exists $Y_{i} \subseteq X_{i}$ with $\mu_{i}\left(Y_{i} \mid t_{i}\right)=1$ satisfying the following: for each $x_{i} \in Y_{i}$ and $\varepsilon>0$, there is a neighborhood $V_{x_{i}}$ of $x_{i}$ such that for every $y_{i} \in V_{x_{i}}$, there is a neighborhood $V_{x_{-i}}$ of $x_{-i}$ such that

$$
u_{i}\left(t,\left(y_{i}, y_{-i}\right)\right)<u_{i}\left(t,\left(x_{i}, y_{-i}\right)\right)+\varepsilon, \quad \text { for all } y_{-i} \in V_{x_{-i}} .
$$

Generic entire payoff security and generic local equi-upper semicontinuity combined imply strong uniform payoff security.

Lemma 3. Suppose that $\Gamma=\left(T_{i}, X_{i}, u_{i}, p\right)_{i=1}^{N}$ is a Bayesian game satisfying generic entire payoff security and generic local equi-upper semicontinuity. Then $\Gamma$ satisfies strong uniform payoff security.

The proof of Lemma 3 is relegated to Subsection A.3.

From Theorem 1 and Lemma 3 one immediately obtains the second main existence result of the paper.

Theorem 2. Suppose that the Bayesian game $\Gamma=\left(T_{i}, X_{i}, u_{i}, p\right)_{i=1}^{N}$ satisfies generic entire payoff security and generic local equi-upper semicontinuity. Suppose further that for each $t \in T$, the map $\sum_{i=1}^{N} u_{i}(t, \cdot): X \rightarrow \mathbb{R}$ is upper semicontinuous and $p$ is absolutely continuous with respect to $p_{1} \otimes \cdots \otimes p_{N}$. Then $\Gamma$ possesses a perfect Bayes-Nash equilibrium.

\subsection{The special case of complete information games}

In this subsection we state the main existence results in the absence of incomplete information (i.e., when type spaces are singletons), obtaining Theorem 2 and Corollary 1 in Carbonell-Nicolau [9] as special cases of Theorem 1 and Theorem 2.

Definition 15. The mixed extension of a compact, metric, Borel game $G=\left(Z_{i}, g_{i}\right)_{i=1}^{N}$ is the normal-form game $\boldsymbol{G}:=\left(\Delta\left(Z_{i}\right), G_{i}\right)_{i=1}^{N}$, where for each $i, G_{i}: \times_{j=1}^{N} \Delta\left(Z_{j}\right) \rightarrow \mathbb{R}$ is defined by

$$
G_{i}\left(\sigma_{1}, \ldots, \sigma_{N}\right):=\int_{Z} g_{i}(z)\left[\sigma_{1} \otimes \cdots \otimes \sigma_{N}\right](d z) .
$$

Suppose that $G=\left(Z_{i}, g_{i}\right)_{i=1}^{N}$ is a compact, metric, Borel game. For each $i$, let $\widehat{\Delta}\left(Z_{i}\right)$ be the set of all strictly positive members of $\Delta\left(Z_{i}\right)$, i.e., the set of all $\sigma_{i} \in \Delta\left(Z_{i}\right)$ such that $\sigma_{i}(V)>0$ for every nonempty open set $V$ in $Z_{i}$.

Given $\alpha=\left(\alpha_{1}, \ldots, \alpha_{N}\right) \in[0,1)^{N}$ and $\mu=\left(\mu_{1}, \ldots, \mu_{N}\right) \in \times{ }_{i=1}^{N} \widehat{\Delta}\left(Z_{i}\right)$, define the normal-form game

$$
G^{(\alpha, \mu)}:=\left(\Delta\left(Z_{i}\right), G_{i}^{(\alpha, \mu)}\right)_{i=1}^{N},
$$

where $G_{i}^{(\alpha, \mu)}: \times_{j=1}^{N} \Delta\left(Z_{j}\right) \rightarrow \mathbb{R}$ is defined by

$$
G_{i}^{(\alpha, \mu)}\left(\sigma_{1}, \ldots, \sigma_{N}\right):=G_{i}\left(\left(1-\alpha_{1}\right) \sigma+\alpha_{1} \mu_{1}, \ldots,\left(1-\alpha_{N}\right) \sigma_{N}+\alpha_{N} \mu_{N}\right) .
$$


Definition 16. Suppose that $G=\left(Z_{i}, g_{i}\right)_{i=1}^{N}$ is a compact, metric, Borel game. A Nash equilibrium $\sigma$ of the mixed extension $\boldsymbol{G}=\left(\Delta\left(Z_{i}\right), G_{i}\right)_{i=1}^{N}$ is perfect if there exist sequences $\left(\alpha^{n}\right),\left(\mu^{n}\right)$, and $\left(\sigma^{n}\right)$ such that the following holds for each $n: \alpha^{n} \in(0,1)^{N}, \mu^{n} \in \times{ }_{i=1}^{N} \widehat{\Delta}\left(Z_{i}\right)$, and $\sigma^{n}$ is a Nash equilibrium of the game $G^{\left(\alpha^{n}, \mu^{n}\right)}$ defined in (3), and in addition $\alpha^{n} \rightarrow 0$ and $\sigma^{n} \underset{w}{\longrightarrow} \sigma$.

In the special case of complete information games, Definitions 10-14 can be more simply stated as follows.

Definition 17. A compact, metric, Borel game $\left(Z_{i}, g_{i}\right)_{i=1}^{N}$ satisfies strong uniform payoff security if there exists $\mu=\left(\mu_{1}, \ldots, \mu_{N}\right) \in \widehat{\mathscr{D}}$ such that for each $i$ and $\varepsilon>0$ there is a sequence $\left(f^{k}\right)$ of $\left(\mathscr{B}\left(X_{i}\right), \mathscr{B}\left(X_{i}\right)\right)$-measurable maps $f^{k}: X_{i} \rightarrow X_{i}$ satisfying the following:

(a) For each $k$ and $x \in Z$, there exists a neighborhood $V_{x_{-i}}$ of $x_{-i}$ such that

$$
g_{i}\left(f^{k}\left(x_{i}\right), y_{-i}\right)>g_{i}(x)-\varepsilon, \quad \text { for all } y_{-i} \in V_{x_{-i}} .
$$

(b) For each $x_{-i} \in X_{-i}$, there is a subset $Y_{i}$ of $X_{i}$ with $\mu_{i}\left(Y_{i}\right)=1$ satisfying the following: for each $x_{i} \in Y_{i}$, there exists $K$ such that for all $k \geq K$, there is a neighborhood $V_{x_{-i}}^{\prime}$ of $x_{-i}$ such that

$$
g_{i}\left(f^{k}\left(x_{i}\right), y_{-i}\right)<u_{i}\left(x_{i}, y_{-i}\right)+\varepsilon, \text { for all } y_{-i} \in V_{x_{-i}}^{\prime} .
$$

Definition 18. Suppose that $G=\left(Z_{i}, g_{i}\right)_{i=1}^{N}$ is a metric game and let $Y_{1} \subseteq Z_{1}, \ldots, Y_{N} \subseteq Z_{N}$. The game $G$ is said to satisfy entire payoff security over $\times{ }_{i=1}^{N} Y_{i}$ if for each $i, \varepsilon>0$, and $x_{i} \in Y_{i}$, and for every neighborhood $V_{x_{i}}$ of $x_{i}$, there exist $y_{i} \in V_{x_{i}}$ and a neighborhood $V_{x_{i}}^{\prime}$ of $x_{i}$ such that for every $z \in Z$, there is a neighborhood $V_{z_{-i}}$ of $z_{-i}$ such that

$$
g_{i}\left(y_{i}, y_{-i}\right)>g_{i}\left(z_{i}^{\prime}, z_{-i}\right)-\varepsilon, \quad \text { for all }\left(z_{i}^{\prime}, y_{-i}\right) \in V_{x_{i}}^{\prime} \times V_{z_{-i}} .
$$

Definition 19. Suppose that $G=\left(Z_{i}, g_{i}\right)_{i=1}^{N}$ is a metric game and let $Y_{1} \subseteq Z_{1}, \ldots, Y_{N} \subseteq Z_{N}$. The game $G$ is said to satisfy generic entire payoff security over $\times{ }_{i=1}^{N} Y_{i}$ if there exist $Z_{1}^{\prime} \subseteq Y_{1}, \ldots, Z_{N}^{\prime} \subseteq Y_{N}$ such that $Y_{i} \backslash Z_{i}^{\prime}$ is countable for each $i, \Gamma$ is entirely payoff secure over $\times_{i=1}^{N} Z_{i}^{\prime}$, and the following condition is satisfied: for each $i, \varepsilon>0$, and $x_{i} \in Y_{i} \backslash Z_{i}^{\prime}$, there exists $y_{i} \in Z_{i}$ such that for every $z \in Z$, there is a neighborhood $V_{z_{-i}}$ of $z_{-i}$ such that

$$
g_{i}\left(y_{i}, y_{-i}\right)>g_{i}(z)-\varepsilon, \text { for all } y_{-i} \in V_{z_{-i}} \text {. }
$$

Definition 20. A metric game $\left(Z_{i}, g_{i}\right)_{i=1}^{N}$ said to satisfy generic entire payoff security if it is entirely payoff secure over $\times{ }_{i=1}^{N} K_{i}$ and generically entirely payoff secure over $\times{ }_{i=1}^{N} A_{i}$.

Suppose that $\left(Z_{i}, g_{i}\right)_{i=1}^{N}$ is a compact, metric, Borel game. Recall that $Z_{i}$ can be written as a disjoint union $A_{i} \cup K_{i}$, where $A_{i}$ is closed and dense in itself (i.e., with no isolated points) and $K_{i}$ is countable. Recall that the set of all strictly positive mixed strategies in $\Delta\left(Z_{i}\right)$ is denoted by $\widehat{\Delta}\left(Z_{i}\right)$. Let $\widetilde{\Delta}\left(Z_{i}\right)$ be the set of members $\sigma_{i}$ of $\Delta\left(Z_{i}\right)$ such that $\sigma_{i}\left(\left\{x_{i}\right\}\right)=0$ and $\sigma_{i}\left(N_{\epsilon}\left(x_{i}\right)\right)>0$ for every $x_{i} \in A_{i}$ and $\epsilon>0$ (where $N_{\epsilon}\left(x_{i}\right)$ denotes the $\epsilon$-neighborhood of $x_{i}$ ), and $\sigma_{i}\left(\left\{x_{i}\right\}\right)>0$ for every $x_{i} \in K_{i}$.

Definition 21. A compact, metric, Borel game $\left(Z_{i}, g_{i}\right)_{i=1}^{N}$ satisfies generic local equi-upper semicontinuity if there exists $\mu=\left(\mu_{1}, \ldots, \mu_{N}\right) \in \widetilde{\Delta}\left(Z_{i}\right)$ such that for each $i$ and $x_{-i} \in Z_{-i}$, there exists $Y_{i} \subseteq Z_{i}$ with $\mu_{i}\left(Y_{i}\right)=1$ satisfying the following: for each $x_{i} \in Y_{i}$ and $\varepsilon>0$, there is a neighborhood $V_{x_{i}}$ of $x_{i}$ such that for every $y_{i} \in V_{x_{i}}$, there is a neighborhood $V_{x_{-i}}$ of $x_{-i}$ such that

$$
g_{i}\left(y_{i}, y_{-i}\right)<g_{i}\left(x_{i}, y_{-i}\right)+\varepsilon, \quad \text { for all } y_{-i} \in V_{x_{-i}} \text {. }
$$


The following corollaries follow immediately from the main existence results.

Corollary 1 (to Theorem 1). Suppose that the compact, metric, Borel game $G=\left(Z_{i}, g_{i}\right)_{i=1}^{N}$ satisfies strong uniform payoff security and that the $\operatorname{map} \sum_{i=1}^{N} g_{i}(\cdot): Z \rightarrow \mathbb{R}$ is upper semicontinuous. Then $G$ possesses a perfect Nash equilibrium.

Corollary 2 (to Theorem 2). Suppose that the compact, metric, Borel game $G=\left(Z_{i}, g_{i}\right)_{i=1}^{N}$ satisfies generic entire payoff security and generic local equi-upper semicontinuity. If the map $\sum_{i=1}^{N} g_{i}(\cdot): Z \rightarrow \mathbb{R}$ is upper semicontinuous, then $G$ possesses a perfect Nash equilibrium.

\section{Application to all-pay auctions}

This section illustrates the machinery developed in Section 3 in the context of all-pay auctions. We confine attention to a generalized version of the war of attrition considered in Krishna and Morgan [18], but the existence result presented here extends to other all-pay auctions. An existence result is obtained, using Theorem 1 , for the war of attrition with common values and interdependent types.

There are $N$ bidders competing for a single indivisible object. After learning their types, the players simultaneously submit a sealed bid $b_{i}$ from a closed and bounded subinterval $B_{i}:=[\underline{b}, \bar{b}]$ of $\mathbb{R}_{+}($where $\underline{b}<\bar{b})$. Each $B_{i}$ is endowed with the usual relative Euclidean metric, and the Cartesian product $B:=\times_{i=1}^{N} B_{i}$ is equipped with the corresponding supremum metric. Let $T_{1}, \ldots, T_{N}$ be the type spaces (each $T_{i}$ is a compact, metric type space). The highest bidder wins the object and ties are broken via an equal probability rule. If player $i$ wins the object when Nature chooses a type profile $t=\left(t_{1}, \ldots, t_{N}\right) \in T$ and when the profile of bids chosen by the players is $b=\left(b_{1}, \ldots, b_{N}\right) \in B$, then player $i$ 's payoff is given by $v(t)-\max _{j \neq i} b_{j}$, where $v(t) \geq 0$ represents the value of the object in state $t$ and $\max _{j \neq i} b_{j}$ is the second highest bid in the action profile $b$. All the other players $j \neq i$ obtain a payoff of $h_{j}(t, b)$. The common prior over type profiles in $T$ is represented by a probability measure $p$ on $(T, \mathscr{B}(T)$ ), assumed absolutely continuous with respect to the product of its marginal probability measures, $p_{1} \otimes \cdots \otimes p_{N}$.

Bidder $i$ 's expected payoff at $t=\left(t_{1}, \ldots, t_{N}\right) \in T$ and $b=\left(b_{1}, \ldots, b_{N}\right) \in B$ is given by

$$
u_{i}(t, b):= \begin{cases}h_{i}(t, b) & \text { if } b_{i}<\max _{j} b_{j}, \\ \frac{v(t)}{\#\left\{j: b_{j}=\max _{\iota} b_{l}\right\}}-\max _{j \neq i} b_{j} & \text { if } b_{i}=\max _{j} b_{j} .\end{cases}
$$

Here, the map $v: T \rightarrow \mathbb{R}$ is assumed bounded and $(\mathscr{B}(T), \mathscr{B}(\mathbb{R}))$-measurable, and the maps $h_{i}: T \times B \rightarrow \mathbb{R}$ are bounded and $(\mathscr{B}(T \times B), \mathscr{B}(\mathbb{R}))$-measurable and satisfy the following: for each $i$, the family $\left\{h_{i}(t, \cdot): t \in T\right\}$ is equicontinuous on $B$ and $h_{i}(t, b)=-\max _{j \neq i} b_{j}$ whenever $t \in T$ and $b \in B$ satisfies $b_{i}=\max _{j} b_{j}$. In particular, if $h_{i}(t, b)=-b_{i}$ whenever $b_{i}<\max _{j} b_{j}$ (and if one makes additional assumptions on the affiliation of types) one obtains the war of attrition game considered in Krishna and Morgan [18].

The associated Bayesian game is

$$
\Gamma:=\left(T_{i}, B_{i}, u_{i}, p\right)_{i=1}^{N} .
$$

Lemma 4. The game $\Gamma$ defined in (4) satisfies strong uniform payoff security.

Proof. Let $\mu=\left(\mu_{1}, \ldots, \mu_{N}\right) \in \widehat{\mathscr{D}}$ be such that for each $i$ and $t_{i} \in T_{i}, \mu_{i}\left(\cdot \mid t_{i}\right)$ is the normalized Lebesgue measure over $B_{i}$.

Fix $i$ and $\varepsilon>0$. Because $\left\{h_{i}(t, \cdot): t \in T\right\}$ is equicontinuous on the $\operatorname{compact} \operatorname{set} B,\left\{h_{i}(t, \cdot): t \in T\right\}$ is uniformly equicontinuous on $B$. Therefore, there exists $\delta>0$ such that

$$
\left|h_{i}(t, b)-h_{i}\left(t, b^{\prime}\right)\right|<\varepsilon, \quad \text { for all } t \in T \text { and }\left(b, b^{\prime}\right) \in B \times B \text { with } d\left(b, b^{\prime}\right)<\delta,
$$


where $d$ is a compatible metric on $B$.

For each $k$, define $f^{k}: B_{i} \rightarrow B_{i}$ as follows: $f^{k}\left(b_{i}\right):=\frac{1}{k} \bar{b}+\left(1-\frac{1}{k}\right) b_{i}$. Let $k^{*}>\frac{\bar{b}-\underline{b}}{\delta}$ and observe that for $k \geq k^{*}$ and $b_{i} \in B_{i}$,

$$
f^{k}\left(b_{i}\right)-b_{i}=\frac{1}{k}\left(\bar{b}-b_{i}\right) \leq \frac{1}{k}(\bar{b}-\underline{b})<\delta .
$$

Fix $k \geq k^{*}$ and $(t, b) \in T \times B$. We consider three cases:

Case 1. $b_{i}=\max _{j} b_{j}<\bar{b}$. Let $V_{b_{-i}}$ be a neighborhood of $b_{-i}$ contained in $N_{\varepsilon}\left(b_{-i}\right)$ such that $\max _{j \neq i} b_{j}^{\prime}<f^{k}\left(b_{i}\right)$ for all $b_{-i}^{\prime} \in V_{b_{-i}}$, and pick any $b_{-i}^{\prime} \in V_{b_{-i}}$. Then

$$
\begin{aligned}
u_{i}\left(t,\left(f^{k}\left(b_{i}\right), b_{-i}^{\prime}\right)\right)=v(t)-\max _{j} b_{j}^{\prime} & \geq \frac{v(t)}{\#\left\{j: b_{j}=\max _{\iota} b_{l}\right\}}-\max _{j} b_{j}^{\prime} \\
& >\frac{v(t)}{\#\left\{j: b_{j}=\max _{\iota} b_{l}\right\}}-\max _{j} b_{j}-\varepsilon=u_{i}(t, b)-\varepsilon .
\end{aligned}
$$

Case 2. $b_{i}=\max _{j} b_{j}=\bar{b}$. Let $V_{b_{-i}}^{\prime}$ be a neighborhood of $b_{-i}$ contained in $N_{\varepsilon}\left(b_{-i}\right)$ such that $b_{j}^{\prime}<\bar{b}$ for each $j \neq i$ whenever $b_{-i}^{\prime} \in V_{b_{-i}}^{\prime}$ and $b_{j}<\bar{b}$. For any $b_{-i}^{\prime} \in V_{b_{-i}}^{\prime}$,

$$
\begin{aligned}
u_{i}\left(t,\left(f^{k}\left(b_{i}\right), b_{-i}^{\prime}\right)\right) & =u_{i}\left(t,\left(b_{i}, b_{-i}^{\prime}\right)\right)=\frac{v(t)}{1+\#\left\{j: b_{j}^{\prime}=\max _{l} b_{l}^{\prime}\right\}}-\max _{j} b_{j}^{\prime} \\
& >\frac{v(t)}{\#\left\{j: b_{j}=\max _{\iota} b_{l}\right\}}-\max _{j \neq i} b_{j}-\varepsilon=u_{i}(t, b)-\varepsilon .
\end{aligned}
$$

Case 3. $b_{i}<\max _{j} b_{j}$. Choose $b_{-i}^{\prime} \in N_{\delta}\left(b_{-i}\right)$. If $f^{k}\left(b_{i}\right) \geq \max _{j} b_{j}^{\prime}$, then

$$
u_{i}\left(t,\left(f^{k}\left(b_{i}\right), b_{-i}^{\prime}\right)\right) \geq-\max _{j} b_{j}^{\prime}=h_{i}\left(t,\left(f^{k}\left(b_{i}\right), b_{-i}^{\prime}\right)\right)>h_{i}(t, b)-\varepsilon=u_{i}(t, b)-\varepsilon .
$$

If $f^{k}\left(b_{i}\right)<\max _{j} b_{j}^{\prime}$, then

$$
u_{i}\left(t,\left(f^{k}\left(b_{i}\right), b_{-i}^{\prime}\right)\right)=h_{i}\left(t,\left(f^{k}\left(b_{i}\right), b_{-i}^{\prime}\right)\right)>h_{i}(t, b)-\varepsilon=u_{i}(t, b)-\varepsilon .
$$

This establishes item (a) of Definition 10 for $\Gamma$. To see that item (b) holds, fix $\left(t, b_{-i}\right) \in$ $T \times B_{-i}$ and choose $b_{i} \in B_{i}$ with $b_{i} \neq \max _{j \neq i} b_{j}$. If $b_{i}>\max _{j \neq i} b_{j}$, then for each $k$ and for $V_{b_{-i}}$ a neighborhood of $b_{-i}$ such that $b_{j}^{\prime}<b_{i}$ for each $j \neq i$ whenever $b_{-i}^{\prime} \in V_{b_{-i}}$,

$$
u_{i}\left(t,\left(f^{k}\left(b_{i}\right), b_{-i}^{\prime}\right)\right)=v(t)-\max _{j} b_{j}^{\prime}=u_{i}\left(t,\left(b_{i}, b_{-i}^{\prime}\right)\right)<u_{i}\left(t,\left(b_{i}, b_{-i}^{\prime}\right)\right)+\varepsilon, \quad \text { for all } b_{-i}^{\prime} \in V_{b_{-i}} .
$$

If $b_{i}<\max _{j \neq i} b_{j}$, there exists $K$ such that for $k \geq K$ one has $f^{k}\left(b_{i}\right)<\max _{j \neq i} b_{j}-\beta$ for some $\beta>0$ and $f^{k}\left(b_{i}\right)-b_{i}<\delta$, and one can choose a neighborhood $V_{b_{-i}}^{\prime}$ of $b_{-i}$ such that for all $b_{-i}^{\prime} \in V_{b_{-i}}^{\prime}, \max _{j} b_{j}^{\prime}>f^{k}\left(b_{i}\right)>b_{i}$. Then, for $k \geq K$,

$u_{i}\left(t,\left(f^{k}\left(b_{i}\right), b_{-i}^{\prime}\right)\right)=h_{i}\left(t,\left(f^{k}\left(b_{i}\right), b_{-i}^{\prime}\right)\right)<h_{i}\left(t,\left(b_{i}, b_{-i}^{\prime}\right)\right)+\varepsilon=u_{i}\left(t,\left(b_{i}, b_{-i}^{\prime}\right)\right)+\varepsilon, \quad$ for all $b_{-i}^{\prime} \in V_{b_{-i}}$.

This establishes item (b) of Definition 10.

Theorem 3. The game $\Gamma$ defined in (4) possesses a perfect Bayes-Nash equilibrium. 
Proof. In view of Lemma 4, the assertion is an immediate consequence of Theorem 1 once one observes that for each $t \in T$, the map $\sum_{i=1}^{N} u_{i}(t, \cdot): B \rightarrow \mathbb{R}$ is upper semicontinuous (in fact, continuous). The continuity of this sum follows from the fact that, for every $(t, b) \in T \times B$,

$$
\sum_{i=1}^{N} u_{i}(t, b)=v(t)-\#\left\{i: b_{i}=\max _{\iota} b_{\iota}\right\} b^{*}+\sum_{i: b_{i}<\max _{\iota} b_{\iota}} h_{i}(t, b),
$$

where $b^{*}$ represents the second highest bid in $b$, together with the equicontinuity of $\left\{h_{i}(t, \cdot): t \in T\right\}$ on $B$, for each $i$, and the condition that, for each $i, h_{i}(t, b)=-\max _{j \neq i} b_{j}$ whenever $b_{i}=\max _{j} b_{j}$.

\section{A Proofs of Lemma 1, Lemma 2, and Lemma 3}

\section{A.1 Proof of Lemma 1}

Prior to proving Lemma 1 we state and prove the following preliminary result.

Lemma 5. Suppose that the Bayesian game $\Gamma=\left(T_{i}, X_{i}, u_{i}, p\right)_{i=1}^{N}$ satisfies strong uniform payoff security. Suppose that $p$ is absolutely continuous with respect to $p_{1} \otimes \cdots \otimes p_{N}$. Then there exists $\left(\mu_{1}, \ldots, \mu_{N}\right) \in \widehat{\mathscr{D}}$ such that for each $i$ and $\varepsilon>0$, there is a sequence $\left(f^{k}\right)$ of $\left(\mathscr{B}\left(X_{i}\right), \mathscr{B}\left(X_{i}\right)\right)$ measurable maps $f^{k}: X_{i} \rightarrow X_{i}$ satisfying the following:

- For each $\left(t_{i}, x_{i}\right) \in T_{i} \times X_{i}, \sigma_{-i} \in \mathscr{D}_{-i}$, and $k$, there is a neighborhood $V_{\sigma_{-i}}$ of $\sigma_{-i}$ such that

$$
\begin{aligned}
& \int_{T_{-i}} \int_{X_{-i}}\left[u_{i}\left(t,\left(f^{k}\left(x_{i}\right), x_{-i}\right)\right) g(t)\right]\left[\underset{j \neq i}{\otimes} \sigma_{j}^{\prime}\left(\cdot \mid t_{j}\right)\right]\left(d x_{-i}\right)\left[\underset{j \neq i}{\otimes} p_{j}\right]\left(d t_{-i}\right) \\
& >\int_{T_{-i}} \int_{X_{-i}}\left[u_{i}(t, x) g(t)\right]\left[\underset{j \neq i}{\otimes} \sigma_{j}\left(\cdot \mid t_{j}\right)\right]\left(d x_{-i}\right)\left[\underset{j \neq i}{\otimes} p_{j}\right]\left(d t_{-i}\right)-\varepsilon, \text { for all } \sigma_{-i}^{\prime} \in V_{\sigma_{-i}},
\end{aligned}
$$

where $g$ is a density of $p$ with respect to $p_{1} \otimes \cdots \otimes p_{N}$.

- For each $\sigma_{-i} \in \mathscr{D}_{-i}$, there exists $K$ such that for each $k \geq K$, there is a neighborhood $V_{\sigma_{-i}}^{\prime}$ of $\sigma_{-i}$ such that

$$
U_{i}\left(\mu_{i}^{k}, \sigma_{-i}^{\prime}\right)<U_{i}\left(\mu_{i}, \sigma_{-i}^{\prime}\right)+\varepsilon, \text { for all } \sigma_{-i}^{\prime} \in V_{\sigma_{-i}}^{\prime}
$$

where $\mu_{i}^{k}\left(\cdot \mid t_{i}\right)$ is defined by

$$
\mu_{i}^{k}\left(B \mid t_{i}\right):=\mu_{i}\left(f^{k^{-1}}(B) \mid t_{i}\right){ }^{1}
$$

Proof. Strong uniform payoff security gives $\mu=\left(\mu_{1}, \ldots, \mu_{N}\right) \in \widehat{\mathscr{D}}$ such that for each $i$ and $\varepsilon>0$ there is a sequence $\left(f_{(i, \varepsilon)}^{k}\right)_{k=1}^{\infty}$ of $\left(\mathscr{B}\left(X_{i}\right), \mathscr{B}\left(X_{i}\right)\right)$-measurable maps $f_{(i, \varepsilon)}^{k}: X_{i} \rightarrow X_{i}$ satisfying the following:

\footnotetext{
${ }^{1}$ For $t_{i} \in T_{i}$, it is clear that $\mu_{i}^{k}\left(B \mid t_{i}\right) \in[0,1]$ for each $B \in \mathscr{B}\left(X_{i}\right)$. In addition, $\mu_{i}^{k}\left(X_{i} \mid t_{i}\right)=\mu_{i}\left(X_{i} \mid t_{i}\right)=1$, and, given a countable collection $\left(B^{l}\right)_{l=1}^{\infty}$ of pairwise disjoint sets in $\mathscr{B}\left(X_{i}\right)$,

$$
\mu_{i}^{k}\left(\bigcup_{l=1}^{\infty} B^{l} \mid t_{i}\right)=\mu_{i}\left(f^{k^{-1}}\left(\bigcup_{l=1}^{\infty} B^{l}\right) \mid t_{i}\right)=\mu_{i}\left(\bigcup_{l=1}^{\infty} f^{k^{-1}}\left(B^{l}\right) \mid t_{i}\right)=\sum_{l=1}^{\infty} \mu_{i}\left(f^{k^{-1}}\left(B^{l}\right) \mid t_{i}\right)=\sum_{l=1}^{\infty} \mu_{i}^{k}\left(B^{l} \mid t_{i}\right)
$$

Thus, $\mu_{i}^{k}\left(\cdot \mid t_{i}\right) \in \Delta\left(X_{i}\right)$ for each $t_{i} \in T_{i}$. Moreover, given $B \in \mathscr{B}\left(X_{i}\right)$ we have $f^{k^{-1}}(B) \in \mathscr{B}\left(X_{i}\right)$, and so the map $t_{i} \in T_{i} \mapsto \mu_{i}\left(f^{k^{-1}}(B) \mid t_{i}\right)$ is $\left(\mathscr{B}\left(T_{i}\right), \mathscr{B}([0,1])\right)$-measurable by Proposition 7.26 in Bertsekas and Shreve [6]. Hence, the map $t_{i} \in T_{i} \mapsto \mu_{i}^{k}\left(B \mid t_{i}\right)$ is $\left(\mathscr{B}\left(T_{i}\right), \mathscr{B}([0,1])\right)$-measurable and so (again by Proposition 7.26 in [6]) the map $\mu_{i}^{k}: T_{i} \rightarrow \Delta\left(X_{i}\right)$ is $\left(\mathscr{B}\left(T_{i}\right), \mathscr{B}\left(\Delta\left(X_{i}\right)\right)\right)$-measurable.
} 
(a) For each $k$ and $(t, x) \in T \times X$, there exists a neighborhood $V_{x_{-i}}$ of $x_{-i}$ such that

$$
u_{i}\left(t,\left(f_{(i, \varepsilon)}^{k}\left(x_{i}\right), y_{-i}\right)\right) g(t) \geq\left[u_{i}(t, x)-\varepsilon\right] g(t), \quad \text { for all } y_{-i} \in V_{x_{-i}} .
$$

(b) For each $\left(t, x_{-i}\right) \in T \times X_{-i}$, there is a subset $Y_{\left(i, \varepsilon, t, x_{-i}\right)}$ of $X_{i}$ with $\mu_{i}\left(Y_{\left(i, \varepsilon, t, x_{-i}\right)} \mid t_{i}\right)=1$ satisfying the following: for each $x_{i} \in Y_{\left(i, \varepsilon, t, x_{-i}\right)}$, there exists $K_{(i, \varepsilon, t, x)}$ such that for all $k \geq K_{(i, \varepsilon, t, x)}$, there exists $n_{(i, \varepsilon, t, x, k)}$ such that

$$
u_{i}\left(t,\left(f_{(i, \varepsilon)}^{k}\left(x_{i}\right), y_{-i}\right)\right) g(t) \leq\left[u_{i}\left(t,\left(x_{i}, y_{-i}\right)\right)+\varepsilon\right] g(t), \quad \text { for all } y_{-i} \in N_{1 / n_{(i, \varepsilon, t, x, k}}\left(x_{-i}\right) .
$$

Given $\left(i, \varepsilon, t_{-i}, x_{-i}\right)$ and $\{k, n\} \subseteq \mathbb{N}$, define $\xi_{\left(i, \varepsilon, t_{-i}, x_{-i}\right)}^{(k, n)}: T_{i} \times X_{i} \rightarrow \mathbb{R}$ by

$$
\xi_{\left(i, \varepsilon, t_{-i}, x_{-i}\right)}^{(k, n)}\left(t_{i}, x_{i}\right):=\sup _{y_{-i} \in N_{\frac{1}{n}}\left(x_{-i}\right)}\left[\left[u_{i}\left(t,\left(f_{(i, \varepsilon)}^{k}\left(x_{i}\right), y_{-i}\right)\right)-u_{i}\left(t,\left(x_{i}, y_{-i}\right)\right)\right] g(t)\right]
$$

Because $u_{i}$ is $(\mathscr{B}(T \times X), \mathscr{B}(\mathbb{R}))$-measurable (and $T_{i}$ and $X_{i}$ are compact, metric for each $\left.i\right), u_{i}$ is $\left(\mathscr{B}\left(T_{i} \times X_{i}\right) \otimes \mathscr{B}\left(T_{-i} \times X_{-i}\right)\right)$-measurable, hence $\left(\mathscr{B}^{\mu_{i}}\left(T_{i} \times X_{i}\right) \otimes \mathscr{B}\left(T_{-i} \times X_{-i}\right)\right)$-measurable, where $\mathscr{B}^{\mu_{i}}\left(T_{i} \times X_{i}\right)$ denotes the $\mu_{i}$-completion of $\mathscr{B}\left(T_{i} \times X_{i}\right)$. Therefore, since $\mathscr{B}^{\mu_{i}}\left(T_{i} \times X_{i}\right)$ equals its universal completion, it follows from the proof of the Theorem in Carbonell-Nicolau [11] that the map

$$
\left(\left(\tau_{i}, z_{i}\right),\left(\tau_{-i}, z_{-i}\right)\right) \in T_{i} \times X_{i} \times T_{-i} \times X_{-i} \mapsto \sup _{y_{-i} \in N_{\frac{1}{n}}\left(z_{-i}\right)}\left[\left[u_{i}\left(\tau,\left(f_{(i, \varepsilon)}^{k}\left(z_{i}\right), y_{-i}\right)\right)-u_{i}\left(\tau,\left(z_{i}, y_{-i}\right)\right)\right] g(\tau)\right]
$$

is $\left(\mathscr{B}^{\mu_{i}}\left(T_{i} \times X_{i}\right) \otimes \mathscr{B}\left(T_{-i} \times X_{-i}\right), \mathscr{B}(\mathbb{R})\right)$-measurable, and so the map $\xi_{\left(i, \varepsilon, t_{-i}, x_{-i}\right)}^{(k, n)}$ is $\left(\mathscr{B}^{\mu_{i}}\left(T_{i} \times\right.\right.$ $\left.X_{i}\right), \mathscr{B}(\mathbb{R})$ )-measurable (see, e.g., Aliprantis and Border [1, Theorem 4.48]). Consequently, applying Theorem 10.35 in Aliprantis and Border [1], we see that there exist a $\left(\mathscr{B}\left(T_{i} \times\right.\right.$ $\left.\left.X_{i}\right), \mathscr{B}(\mathbb{R})\right)$-measurable map $\widehat{\xi}_{\left(i, \varepsilon, t_{-i}, x_{-i}\right)}^{(k, n)}: T_{i} \times X_{i} \rightarrow \mathbb{R}$ and $\widehat{A} \in \mathscr{B}\left(T_{i} \times X_{i}\right)$ such that

$$
\mu_{i}(\widehat{A})=0 \quad \text { and } \quad \widehat{\xi}_{\left(i, \varepsilon, t_{-i}, x_{-i}\right)}^{(k, n)}\left(t_{i}, x_{i}\right)=\xi_{\left(i, \varepsilon, t_{-i}, x_{-i}\right)}^{(k, n)}\left(t_{i}, x_{i}\right) \text { for all }\left(t_{i}, x_{i}\right) \in\left(T_{i} \times X_{i}\right) \backslash \widehat{A} .
$$

Let $\xi_{\left(i, \varepsilon, t_{-i}, x_{-i}\right)}: T_{i} \times X_{i} \rightarrow \mathbb{R}$ be defined by

$$
\xi_{\left(i, \varepsilon, t_{-i}, x_{-i}\right)}\left(t_{i}, x_{i}\right):=\lim _{k \rightarrow \infty}\left[\lim _{n \rightarrow \infty} \xi_{\left(i, \varepsilon, t_{-i}, x_{-i}\right)}^{(k, n)}\left(t_{i}, x_{i}\right)\right] .
$$

Then there exists a sequence $\left(n_{k}\right)$ such that

$$
\xi_{\left(i, \varepsilon, t_{-i}, x_{-i}\right)}\left(t_{i}, x_{i}\right):=\lim _{k \rightarrow \infty} \xi_{\left(i, \varepsilon, t_{-i}, x_{-i}\right)}^{\left(k, n_{k}\right)}\left(t_{i}, x_{i}\right)
$$

By Egorov's Theorem (e.g., see Dudley [17, Theorem 7.5.1]), there exists $A \subseteq\left(T_{i} \times X_{i}\right) \backslash \widehat{A}$ such that

$$
\mu_{i}(A)\left[\sup _{\left((\tau, z),\left(\tau^{\prime}, z^{\prime}\right)\right) \in T \times X \times T \times X}\left[u_{i}(\tau, z)-u_{i}\left(\tau^{\prime}, z^{\prime}\right)\right]\right]<\varepsilon
$$

and $\xi_{\left(i, \varepsilon, t_{-i}, x_{-i}\right)}^{\left(k, n_{k}\right)}$ converges to $\xi_{\left(i, \varepsilon, t_{-i}, x_{-i}\right)}$ uniformly on $\left(T_{i} \times X_{i}\right) \backslash(\widehat{A} \cup A)$. Therefore, there exists $\bar{k}$ such that for all $k \geq \bar{k}$ and $\left(t_{i}, x_{i}\right) \in\left(T_{i} \times X_{i}\right) \backslash(\widehat{A} \cup A)$,

$$
\left|\xi_{\left(i, \varepsilon, t_{-i}, x_{-i}\right)}^{\left(k, n_{k}\right)}\left(t_{i}, x_{i}\right)-\xi_{\left(i, \varepsilon, t_{-i}, x_{-i}\right)}\left(t_{i}, x_{i}\right)\right|<\varepsilon .
$$

In addition, by (b) one has, for $t_{i} \in T_{i}$ and $x_{i} \in Y_{\left(i, \varepsilon, t, x_{-i}\right)}$,

$$
\xi_{\left(i, \varepsilon, t_{-i}, x_{-i}\right)}\left(t_{i}, x_{i}\right) \leq \varepsilon g(t) .
$$


Consequently, for all $k \geq \bar{k}$,

$$
\xi_{\left(i, \varepsilon, t_{-i}, x_{-i}\right)}^{\left(k, n_{k}\right)}\left(t_{i}, x_{i}\right)<\varepsilon+\varepsilon g(t)
$$

for all $\left(t_{i}, x_{i}\right) \in\left(T_{i} \times X_{i}\right) \backslash(\widehat{A} \cup A)$ with $x_{i} \in Y_{\left(i, \varepsilon, t, x_{-i}\right)}$, and so for each $k \geq \bar{k}$,

$$
\sup _{y_{-i} \in N_{\frac{1}{n_{k}}}\left(x_{-i}\right)}\left[\left[u_{i}\left(t,\left(f_{(i, \varepsilon)}^{k}\left(x_{i}\right), y_{-i}\right)\right)-u_{i}\left(t,\left(x_{i}, y_{-i}\right)\right)\right] g(t)\right]<\varepsilon+\varepsilon g(t)
$$

for all $\left(t_{i}, x_{i}\right) \in\left(T_{i} \times X_{i}\right) \backslash(\widehat{A} \cup A)$ with $x_{i} \in Y_{\left(i, \varepsilon, t, x_{-i}\right)}$, whence for each $k \geq \bar{k}$ and $y_{-i} \in N_{1 / n_{k}}\left(x_{-i}\right)$,

$$
\left[u_{i}\left(t,\left(f_{(i, \varepsilon)}^{k}\left(x_{i}\right), y_{-i}\right)\right)-u_{i}\left(t,\left(x_{i}, y_{-i}\right)\right)\right] g(t)<\varepsilon+\varepsilon g(t)
$$

for all $\left(t_{i}, x_{i}\right) \in\left(T_{i} \times X_{i}\right) \backslash(\widehat{A} \cup A)$ with $x_{i} \in Y_{\left(i, \varepsilon, t, x_{-}\right)}$. Hence, in light of (7) and (8), it follows that for each $k \geq \bar{k}$ and $y_{-i} \in N_{1 / n_{k}}\left(x_{-i}\right)$,

$$
\begin{aligned}
& \int_{T_{i} \times X_{i}}\left[u_{i}\left(t,\left(f_{(i, \varepsilon)}^{k}\left(x_{i}\right), y_{-i}\right)\right)-u_{i}\left(t,\left(x_{i}, y_{-i}\right)\right)\right] g(t) \mu_{i}\left(d\left(t_{i}, x_{i}\right)\right) \\
& =\int_{T_{i}} \int_{Y_{\left(i, \varepsilon, t, x_{-i}\right)}}\left[u_{i}\left(t,\left(f_{(i, \varepsilon)}^{k}\left(x_{i}\right), y_{-i}\right)\right)-u_{i}\left(t,\left(x_{i}, y_{-i}\right)\right)\right] g(t) \mu_{i}\left(d x_{i} \mid t_{i}\right) p_{i}\left(d t_{i}\right)<\varepsilon+2 \varepsilon \int_{T_{i}} g(t) p_{i}\left(d t_{i}\right) .
\end{aligned}
$$

We conclude that for given $\left(i, \varepsilon, t_{-i}, x_{-i}\right)$, there exists $\bar{k}_{\left(i, \varepsilon, t_{-i}, x_{-i}\right)}$ such that for each $k \geq$ $\bar{k}_{\left(i, \varepsilon, t_{-i}, x_{-i}\right)}$ and $y_{-i} \in N_{1 / n_{k}}\left(x_{-i}\right)$,

$$
\int_{T_{i} \times X_{i}}\left[u_{i}\left(t,\left(f_{(i, \varepsilon)}^{k}\left(x_{i}\right), y_{-i}\right)\right)-u_{i}\left(t,\left(x_{i}, y_{-i}\right)\right)\right] g(t) \mu_{i}\left(d\left(t_{i}, x_{i}\right)\right)<\varepsilon+2 \varepsilon \int_{T_{i}} g(t) p_{i}\left(d t_{i}\right) .
$$

Next, define $\psi_{(i, \varepsilon)}^{k}: T_{-i} \times X_{-i} \rightarrow \mathbb{R}$ by

$$
\psi_{(i, \varepsilon)}^{k}\left(t_{-i}, x_{-i}\right):=\int_{T_{i} \times X_{i}}\left[u_{i}\left(t,\left(f_{(i, \varepsilon)}^{k}\left(x_{i}\right), x_{-i}\right)\right)-u_{i}\left(t,\left(x_{i}, x_{-i}\right)\right)\right] g(t) \mu_{i}\left(d\left(t_{i}, x_{i}\right)\right)
$$

and $\bar{\psi}_{(i, \varepsilon)}^{k}: T_{-i} \times X_{-i} \rightarrow \mathbb{R}$ by

$$
\bar{\psi}_{(i, \varepsilon)}^{k}\left(t_{-i}, x_{-i}\right):=\lim _{n \rightarrow \infty} \sup _{y_{-i} \in N_{\frac{1}{n}}\left(x_{-i}\right)} \psi_{(i, \varepsilon)}^{k}\left(t_{-i}, y_{-i}\right) .
$$

Fix $(i, \varepsilon)$ and $\sigma_{-i} \in \mathscr{D}_{-i}$. Because $\psi_{(i, \eta)}^{k}$ is $\left(\mathscr{B}\left(T_{-i}\right) \otimes \mathscr{B}\left(X_{-i}\right), \mathscr{B}(\mathbb{R})\right)$-measurable, it follows from the Theorem in Carbonell-Nicolau [11] that $\bar{\psi}_{(i, \eta)}^{k}$ is $\left(\mathscr{B}^{u}\left(T_{-i}\right) \otimes \mathscr{B}\left(X_{-i}\right), \mathscr{B}(\mathbb{R})\right)$ measurable, where $\mathscr{B}^{u}\left(T_{-i}\right)$ denotes the universal completion of $\mathscr{B}\left(T_{-i}\right)$. Consequently, since the map $x_{-i} \in X_{-i} \mapsto \bar{\psi}_{(i, \eta)}^{k}\left(t_{-i}, x_{-i}\right)$ is upper semicontinuous for each $t_{-i} \in T_{-i}$, Corollary 1 in Averna [3] gives a compact set $B \subseteq T_{-i}$ such that

$$
p\left(T_{i} \times\left(T_{-i} \backslash B\right)\right)\left[\sup _{\left((\tau, z),\left(\tau^{\prime}, z^{\prime}\right)\right) \in T \times X \times T \times X}\left[u_{i}(\tau, z)-u_{i}\left(\tau^{\prime}, z^{\prime}\right)\right]\right]<\eta
$$

and $\left.\bar{\psi}_{(i, \eta)}^{k}\right|_{B \times X_{-i}}$ is upper semicontinuous. Hence, letting $\widehat{\psi}_{(i, \eta)}^{k}: T_{-i} \times X_{-i} \rightarrow \mathbb{R}$ be defined by

$$
\widehat{\psi}_{(i, \eta)}^{k}\left(t_{-i}, x_{-i}\right):=\sup _{k^{\prime} \geq k} \bar{\psi}_{(i, \eta)}^{k^{\prime}}\left(t_{-i}, x_{-i}\right)
$$

it follows that $\left.\widehat{\psi}_{(i, \eta)}^{k}\right|_{B \times X_{-i}}$ is $\left(\mathscr{B}\left(B \times X_{-i}\right), \mathscr{B}(\mathbb{R})\right)$-measurable. Now define $\widehat{\psi}_{(i, \eta)}: B \times X_{-i} \rightarrow \mathbb{R}$ by

$$
\widehat{\psi}_{(i, \eta)}\left(t_{-i}, x_{-i}\right):=\lim _{k \rightarrow \infty} \widehat{\psi}_{(i, \eta)}^{k}\left(t_{-i}, x_{-i}\right) .
$$


Applying Egorov's Theorem (e.g., see Dudley [17, Theorem 7.5.1]), there exists $\widehat{B} \subseteq B \times X_{-i}$ such that

$$
\left[\underset{j \neq i}{\otimes} \sigma_{j}\right](\widehat{B})\left[\sup _{\left(t_{-i}, x_{-i}\right) \in B \times X_{-i}} \bar{\psi}_{(i, \eta)}^{k}\left(t_{-i}, x_{-i}\right)\right]<\eta
$$

and $\widehat{\psi}_{(i, \eta)}^{k}$ converges uniformly to $\widehat{\psi}_{(i, \eta)}$ on $\left(B \times X_{-i}\right) \backslash \widehat{B}$, i.e., there exists $\overline{\bar{k}}$ such that for all $k \geq \overline{\bar{k}}$ and $\left(t_{-i}, x_{-i}\right) \in\left(B \times X_{-i}\right) \backslash \widehat{B}$,

$$
\left|\widehat{\psi}_{(i, \eta)}^{k}\left(t_{-i}, x_{-i}\right)-\widehat{\psi}_{(i, \eta)}\left(t_{-i}, x_{-i}\right)\right|<\eta
$$

and because (9) implies

$$
\widehat{\psi}_{(i, \eta)}\left(t_{-i}, x_{-i}\right)<\eta+2 \eta \int_{T_{i}} g(t) p_{i}\left(d t_{i}\right), \quad \text { for all }\left(t_{-i}, x_{-i}\right) \in\left(B \times X_{-i}\right) \backslash \widehat{B},
$$

it follows that for all $k \geq \overline{\bar{k}}$,

$$
\widehat{\psi}_{(i, \eta)}^{k}\left(t_{-i}, x_{-i}\right)<2 \eta+2 \eta \int_{T_{i}} g(t) p_{i}\left(d t_{i}\right), \quad \text { for all }\left(t_{-i}, x_{-i}\right) \in\left(B \times X_{-i}\right) \backslash \widehat{B},
$$

and so for all $k \geq \overline{\bar{k}}$,

$$
\bar{\psi}_{(i, \eta)}^{k}\left(t_{-i}, x_{-i}\right)<2 \eta+2 \eta \int_{T_{i}} g(t) p_{i}\left(d t_{i}\right), \quad \text { for all }\left(t_{-i}, x_{-i}\right) \in\left(B \times X_{-i}\right) \backslash \widehat{B} .
$$

This, together with (11) gives, for all $k \geq \overline{\bar{k}}$,

$$
\int_{B \times X_{-i}} \bar{\psi}_{(i, \eta)}^{k}\left(t_{-i}, x_{-i}\right)\left[\underset{j \neq i}{\otimes} \sigma_{j}\right]\left(d\left(t_{-i}, x_{-i}\right)\right)<5 \eta .
$$

On the other hand, recall that the map $\left.\bar{\psi}_{(i, \eta)}^{k}\right|_{B \times X_{-i}}$ is upper semicontinuous, and so applying Theorem 3.7(viii) of Schäl [25] and Theorem 3.1 of Balder [5], it follows that there is a neighborhood $V_{(i, \eta)}^{k}$ of $\sigma_{-i}$ such that

$$
\begin{aligned}
\int_{B \times X_{-i}} \bar{\psi}_{(i, \eta)}^{k}\left(t_{-i}, x_{-i}\right) & {\left[\underset{j \neq i}{\otimes} \sigma_{j}^{\prime}\right]\left(d\left(t_{-i}, x_{-i}\right)\right) } \\
& <\int_{B \times X_{-i}} \bar{\psi}_{(i, \eta)}^{k}\left(t_{-i}, x_{-i}\right)\left[\underset{j \neq i}{\otimes} \sigma_{j}\right]\left(d\left(t_{-i}, x_{-i}\right)\right)+\frac{\varepsilon}{2}, \quad \text { for all } \sigma_{-i}^{\prime} \in V_{(i, \eta)}^{k} .
\end{aligned}
$$

Consequently, since $\psi_{(i, \eta)}^{k} \leq \bar{\psi}_{(i, \eta)}^{k}$, we see that

$$
\begin{aligned}
\int_{B \times X_{-i}} \psi_{(i, \eta)}^{k}\left(t_{-i}, x_{-i}\right) & {\left[\underset{j \neq i}{\otimes} \sigma_{j}^{\prime}\right]\left(d\left(t_{-i}, x_{-i}\right)\right) } \\
& <\int_{B \times X_{-i}} \bar{\psi}_{(i, \eta)}^{k}\left(t_{-i}, x_{-i}\right)\left[\underset{j \neq i}{\otimes} \sigma_{j}\right]\left(d\left(t_{-i}, x_{-i}\right)\right)+\frac{\varepsilon}{2}, \quad \text { for all } \sigma_{-i}^{\prime} \in V_{(i, \eta)}^{k} .
\end{aligned}
$$

Combining this with (12) gives, for $(i, \eta)$, a $k_{(i, \eta)}$ such that for all $k \geq k_{(i, \eta)}$, there is a neighborhood $V_{(i, \eta)}^{k}$ of $\sigma_{-i}$ such that

$$
\int_{B \times X_{-i}} \psi_{(i, \eta)}^{k}\left(t_{-i}, x_{-i}\right)\left[\underset{j \neq i}{\otimes} \sigma_{j}^{\prime}\right]\left(d\left(t_{-i}, x_{-i}\right)\right)<\frac{\varepsilon}{2}+5 \eta, \quad \text { for all } \sigma_{-i}^{\prime} \in V_{(i, \eta)}^{k} .
$$


Hence, thanks to (10), there is, for given $(i, \eta)$, a $k_{(i, \eta)}$ such that for all $k \geq k_{(i, \eta)}$, there is a neighborhood $V_{(i, \eta)}^{k}$ of $\sigma_{-i}$ such that

$$
\begin{aligned}
& \int_{T_{-i} \times X_{-i}} \psi_{(i, \eta)}^{k}\left(t_{-i}, x_{-i}\right)\left[\underset{j \neq i}{\otimes} \sigma_{j}^{\prime}\right]\left(d\left(t_{-i}, x_{-i}\right)\right) \\
& =\int_{\left(T_{-i} \backslash B\right) \times X_{-i}} \psi_{(i, \eta)}^{k}\left(t_{-i}, x_{-i}\right)\left[\underset{j \neq i}{\otimes} \sigma_{j}^{\prime}\right]\left(d\left(t_{-i}, x_{-i}\right)\right)+\int_{B \times X_{-i}} \psi_{(i, \eta)}^{k}\left(t_{-i}, x_{-i}\right)\left[\underset{j \neq i}{\otimes} \sigma_{j}^{\prime}\right]\left(d\left(t_{-i}, x_{-i}\right)\right) \\
& =\int_{\left(T_{-i} \backslash B\right) \times X_{-i}}\left[\int_{T_{i} \times X_{i}}\left[u_{i}\left(t,\left(f_{(i, \varepsilon)}^{k}\left(x_{i}\right), x_{-i}\right)\right)-u_{i}\left(t,\left(x_{i}, x_{-i}\right)\right)\right] g(t) \mu_{i}\left(d\left(t_{i}, x_{i}\right)\right)\right]\left[\underset{j \neq i}{\otimes} \sigma_{j}^{\prime}\right]\left(d\left(t_{-i}, x_{-i}\right)\right) \\
& +\int_{B \times X_{-i}} \psi_{(i, \eta)}^{k}\left(t_{-i}, x_{-i}\right)\left[\underset{j \neq i}{\otimes} \sigma_{j}^{\prime}\right]\left(d\left(t_{-i}, x_{-i}\right)\right) \\
& =\int_{T_{i} \times\left(T_{-i} \backslash B\right)} \int_{X}\left[u_{i}\left(t,\left(f_{(i, \varepsilon)}^{k}\left(x_{i}\right), x_{-i}\right)\right)-u_{i}\left(t,\left(x_{i}, x_{-i}\right)\right)\right]\left[\mu_{i}\left(\cdot \mid t_{i}\right) \otimes\left[\underset{j \neq i}{\otimes} \sigma^{\prime}\left(\cdot \mid t_{j}\right)\right]\right](d x) p\left(d\left(t_{i}, t_{-i}\right)\right) \\
& \quad+\int_{B \times X_{-i}} \psi_{(i, \eta)}^{k}\left(t_{-i}, x_{-i}\right)\left[\underset{j \neq i}{\otimes} \sigma_{j}^{\prime}\right]\left(d\left(t_{-i}, x_{-i}\right)\right) \\
& \leq p\left(T_{i} \times\left(T_{-i} \backslash B\right)\right)\left[\underset{\left((\tau, z),\left(\tau^{\prime}, z^{\prime}\right)\right) \in T \times X \times T \times X}{\left[u_{i}(\tau, z)-u_{i}\left(\tau^{\prime}, z^{\prime}\right)\right]}\right] \\
& \quad+\int_{B \times X_{-i}} \psi_{(i, \eta)}^{k}\left(t_{-i}, x_{-i}\right)\left[\underset{j \neq i}{\otimes} \sigma_{j}^{\prime}\right]\left(d\left(t_{-i}, x_{-i}\right)\right)
\end{aligned}
$$

Choosing $\eta \in\left(0, \frac{\varepsilon}{12}\right)$ gives $K$ such that for all $k \geq K$, there is a neighborhood $V_{\sigma_{-i}}^{\prime}$ of $\sigma_{-i}$ such that (6) holds.

To prove the first bullet point of the lemma, fix $\left(t_{i}, x_{i}\right) \in T_{i} \times X_{i}, \sigma_{-i} \in \mathscr{D}_{-i}$, and $k$. Define $\zeta_{\left(i, \eta, t_{i}, x_{i}\right)}^{k}: T_{-i} \times X_{-i} \rightarrow \mathbb{R}$ by

$$
\zeta_{\left(i, \eta, t_{i}, x_{i}\right)}^{k}\left(t_{-i}, x_{-i}\right):=\sup _{n \in \mathbb{N}} \inf _{y_{-i} \in N_{\frac{1}{n}}\left(x_{-i}\right)} u_{i}\left(t,\left(f_{(i, \eta)}^{k}\left(x_{i}\right), y_{-i}\right)\right) g(t) .
$$

Because $u_{i}$ is $\left(\mathscr{B}\left(T_{i} \times X_{i}\right) \otimes \mathscr{B}\left(T_{-i} \times X_{-i}\right), \mathscr{B}(\mathbb{R})\right)$-measurable, the map

$$
\left(t_{-i}, x_{-i}\right) \in T_{-i} \times X_{-i} \mapsto u_{i}\left(t,\left(f_{(i, \eta)}^{k}\left(x_{i}\right), x_{-i}\right)\right) g(t)
$$

is $\left(\mathscr{B}\left(T_{-i} \times X_{-i}\right), \mathscr{B}(\mathbb{R})\right)$-measurable (see, e.g., Aliprantis and Border [1, Theorem 4.48]), and hence $\left(\mathscr{B}\left(T_{-i}\right) \otimes \mathscr{B}\left(X_{-i}\right), \mathscr{B}(\mathbb{R})\right)$-measurable. Consequently, applying the Theorem in Carbonell-Nicolau [11], it follows that $\zeta_{\left(i, \eta, t_{i}, x_{i}\right)}^{k}$ is $\left(\mathscr{B}^{u}\left(T_{-i}\right) \otimes \mathscr{B}\left(X_{-i}\right), \mathscr{B}(\mathbb{R})\right)$-measurable, where $\mathscr{B}^{u}\left(T_{-i}\right)$ represents the universal completion of $\mathscr{B}\left(T_{-i}\right)$. Therefore, since the map $x_{-i} \in X_{-i} \mapsto \zeta_{\left(i, \eta, t_{i}, x_{i}\right)}^{k}\left(t_{-i}, x_{-i}\right)$ is lower semicontinuous for every $t_{-i} \in T_{-i}$, Corollary 1 in Averna [3] gives a compact set $C \subseteq T_{-i}$ such that

$$
p\left(T_{i} \times\left(T_{-i} \backslash C\right)\right)\left[\sup _{(\tau, z) \in T \times X} u_{i}(\tau, z)\right]<\eta .
$$

and $\left.\zeta_{\left(i, \eta, t_{i}, x_{i}\right)}^{k}\right|_{C \times X_{-i}}$ is lower semicontinuous. Applying Theorem 3.7(viii) of Schäl [25] and Theorem 3.1 of Balder [5], it follows that there is a neighborhood $V_{\left(i, \eta, t_{i}, x_{i}\right)}^{k}$ of $\sigma_{-i}$ such that

$$
\begin{aligned}
\int_{C \times X_{-i}} \zeta_{\left(i, \eta, t_{i}, x_{i}\right)}^{k}\left(t_{-i}, x_{-i}\right)\left[\underset{j \neq i}{\otimes} \sigma_{j}^{\prime}\right]\left(d\left(t_{-i}, x_{-i}\right)\right) \\
\quad>\int_{C \times X_{-i}} \zeta_{\left(i, \eta, t_{i}, x_{i}\right)}^{k}\left(t_{-i}, x_{-i}\right)\left[\underset{j \neq i}{\otimes} \sigma_{j}\right]\left(d\left(t_{-i}, x_{-i}\right)\right)-\eta, \quad \text { for all } \sigma_{-i}^{\prime} \in V_{\left(i, \eta, t_{i}, x_{i}\right)}^{k} .
\end{aligned}
$$


Consequently, for $\sigma_{-i}^{\prime} \in V_{\left(i, \eta, t_{i}, x_{i}\right)}^{k}$, one has, thanks to (13),

$$
\begin{aligned}
& \int_{T_{-i} \times X_{-i}} \zeta_{\left(i, \eta, t_{i}, x_{i}\right)}^{k}\left(t_{-i}, x_{-i}\right)\left[\underset{j \neq i}{\otimes} \sigma_{j}^{\prime}\right]\left(d\left(t_{-i}, x_{-i}\right)\right) \\
& =\int_{\left(T_{-i} \backslash C\right) \times X_{-i}} \zeta_{\left(i, \eta, t_{i}, x_{i}\right)}^{k}\left(t_{-i}, x_{-i}\right)\left[\underset{j \neq i}{\otimes} \sigma_{j}^{\prime}\right]\left(d\left(t_{-i}, x_{-i}\right)\right)+\int_{C \times X_{-i}} \zeta_{\left(i, \eta, t_{i}, x_{i}\right)}^{k}\left(t_{-i}, x_{-i}\right)\left[\underset{j \neq i}{\otimes} \sigma_{j}^{\prime}\right]\left(d\left(t_{-i}, x_{-i}\right)\right) \\
& =\int_{T_{-i} \backslash C} \int_{X_{-i}} \zeta_{\left(i, \eta, t_{i}, x_{i}\right)}^{k}\left(t_{-i}, x_{-i}\right)\left[\underset{j \neq i}{\otimes} \sigma_{j}^{\prime}\left(\cdot \mid t_{j}\right)\right]\left(d x_{-i}\right)\left[\underset{j \neq i}{\otimes} p_{j}\right]\left(d t_{-i}\right) \\
& +\int_{C \times X_{-i}} \zeta_{\left(i, \eta, t_{i}, x_{i}\right)}^{k}\left(t_{-i}, x_{-i}\right)\left[\underset{j \neq i}{\otimes} \sigma_{j}^{\prime}\right]\left(d\left(t_{-i}, x_{-i}\right)\right) \\
& =\int_{T_{-i} \backslash C} \int_{X_{-i}}\left[\sup _{n \in \mathbb{N}} \inf _{y_{-i} \in N_{\frac{1}{n}}\left(x_{-i}\right)} u_{i}\left(t,\left(f_{(i, \eta)}^{k}\left(x_{i}\right), y_{-i}\right)\right)\right]\left[\underset{j \neq i}{\otimes} \sigma_{j}^{\prime}\left(\cdot \mid t_{j}\right)\right]\left(d x_{-i}\right) p\left(d\left(\tau_{i}, t_{-i}\right)\right) \\
& +\int_{C \times X_{-i}} \zeta_{\left(i, \eta, t_{i}, x_{i}\right)}^{k}\left(t_{-i}, x_{-i}\right)\left[\underset{j \neq i}{\otimes} \sigma_{j}^{\prime}\right]\left(d\left(t_{-i}, x_{-i}\right)\right) \\
& \geq p\left(T_{i} \times\left(T_{-i} \backslash C\right)\right)\left[\inf _{(\tau, z) \in T \times X} u_{i}(\tau, z)\right]+\int_{C \times X_{-i}} \zeta_{\left(i, \eta, t_{i}, x_{i}\right)}^{k}\left(t_{-i}, x_{-i}\right)\left[\underset{j \neq i}{\otimes} \sigma_{j}^{\prime}\right]\left(d\left(t_{-i}, x_{-i}\right)\right) \\
& >\int_{C \times X_{-i}} \zeta_{\left(i, \eta, t_{i}, x_{i}\right)}^{k}\left(t_{-i}, x_{-i}\right)\left[\underset{j \neq i}{\otimes} \sigma_{j}^{\prime}\right]\left(d\left(t_{-i}, x_{-i}\right)\right)-\eta \\
& >\int_{C \times X_{-i}} \zeta_{\left(i, \eta, t_{i}, x_{i}\right)}^{k}\left(t_{-i}, x_{-i}\right)\left[\underset{j \neq i}{\otimes} \sigma_{j}\right]\left(d\left(t_{-i}, x_{-i}\right)\right)-2 \eta \\
& =\int_{T_{-i} \times X_{-i}} \zeta_{\left(i, \eta, t_{i}, x_{i}\right)}^{k}\left(t_{-i}, x_{-i}\right)\left[\underset{j \neq i}{\otimes} \sigma_{j}\right]\left(d\left(t_{-i}, x_{-i}\right)\right)-2 \eta-\int_{\left(T_{-i} \backslash C\right) \times X_{-i}} \zeta_{\left(i, \eta, t_{i}, x_{i}\right)}^{k}\left(t_{-i}, x_{-i}\right)\left[\underset{j \neq i}{\otimes} \sigma_{j}\right]\left(d\left(t_{-i}, x_{-i}\right)\right) \\
& =\int_{T_{-i} \times X_{-i}} \zeta_{\left(i, \eta, t_{i}, x_{i}\right)}^{k}\left(t_{-i}, x_{-i}\right)\left[\underset{j \neq i}{\otimes} \sigma_{j}\right]\left(d\left(t_{-i}, x_{-i}\right)\right)-2 \eta \\
& -\int_{\left(T_{-i} \backslash C\right)} \int_{X_{-i}} \zeta_{\left(i, \eta, t_{i}, x_{i}\right)}^{k}\left(t_{-i}, x_{-i}\right)\left[\underset{j \neq i}{\otimes} \sigma_{j}\left(\cdot \mid t_{j}\right)\right]\left(d x_{-i}\right)\left[\underset{j \neq i}{\otimes} p_{j}\right]\left(d t_{-i}\right) \\
& =\int_{T_{-i} \times X_{-i}} \zeta_{\left(i, \eta, t_{i}, x_{i}\right)}^{k}\left(t_{-i}, x_{-i}\right)\left[\underset{j \neq i}{\otimes} \sigma_{j}\right]\left(d\left(t_{-i}, x_{-i}\right)\right)-2 \eta \\
& -\int_{\left(T_{-i} \backslash C\right)} \int_{X_{-i}}\left[\sup _{n \in \mathbb{N}} \inf _{y_{-i} \in N_{\frac{1}{n}}\left(x_{-i}\right)} u_{i}\left(t,\left(f_{(i, \eta)}^{k}\left(x_{i}\right), y_{-i}\right)\right)\right]\left[\underset{j \neq i}{\otimes} \sigma_{j}\left(\cdot \mid t_{j}\right)\right]\left(d x_{-i}\right) p\left(d\left(\tau_{i}, t_{-i}\right)\right) \\
& >\int_{T_{-i} \times X_{-i}} \zeta_{\left(i, \eta, t_{i}, x_{i}\right)}^{k}\left(t_{-i}, x_{-i}\right)\left[\underset{j \neq i}{\otimes} \sigma_{j}\right]\left(d\left(t_{-i}, x_{-i}\right)\right)-3 \eta .
\end{aligned}
$$

In addition, for every $\left(t_{-i}, x_{-i}\right) \in T_{-i} \times X_{-i}$ one has

$$
u_{i}\left(t,\left(f_{(i, \eta)}^{k}\left(x_{i}\right), y_{-i}\right)\right) g(t) \geq \zeta_{\left(i, \eta, t_{i}, x_{i}\right)}^{k}\left(t_{-i}, x_{-i}\right) \geq\left[u_{i}(t, x)-\eta\right] g(t) .
$$

Combining this with the previous inequalities gives, for all $\sigma_{-i}^{\prime} \in V_{\left(i, \eta, t_{i}, x_{i}\right)}^{k}$,

$$
\begin{aligned}
\int_{T_{-i}} \int_{X_{-i}}\left[u_{i}\left(t,\left(f^{k}\left(x_{i}\right), x_{-i}\right)\right) g(t)\right]\left[\underset{j \neq i}{\otimes} \sigma_{j}^{\prime}\left(\cdot \mid t_{j}\right)\right]\left(d x_{-i}\right)\left[\underset{j \neq i}{\otimes} p_{j}\right]\left(d t_{-i}\right) \\
\geq \int_{T_{-i}} \int_{X_{-i}} \zeta_{\left(i, \eta, t_{i}, x_{i}\right)}^{k}\left(t_{-i}, x_{-i}\right)\left[\underset{j \neq i}{\otimes} \sigma_{j}^{\prime}\left(\cdot \mid t_{j}\right)\right]\left(d x_{-i}\right)\left[\underset{j \neq i}{\otimes} p_{j}\right]\left(d t_{-i}\right) \\
>\int_{T_{-i}} \int_{X_{-i}} \zeta_{\left(i, \eta, t_{i}, x_{i}\right)}^{k}\left(t_{-i}, x_{-i}\right)\left[\underset{j \neq i}{\otimes} \sigma_{j}\left(\cdot \mid t_{j}\right)\right]\left(d x_{-i}\right)\left[\underset{j \neq i}{\otimes} p_{j}\right]\left(d t_{-i}\right)-3 \eta \\
\geq \int_{T_{-i}} \int_{X_{-i}}\left[u_{i}(t, x) g(t)\right]\left[\underset{j \neq i}{\otimes} \sigma_{j}\left(\cdot \mid t_{j}\right)\right]\left(d x_{-i}\right)\left[\underset{j \neq i}{\otimes} p_{j}\right]\left(d t_{-i}\right)-4 \eta .
\end{aligned}
$$


Now choosing a small enough $\eta$ gives the desired inequality in (5).

We are now ready to prove Lemma 1 , which is restated here for the convenience of the reader.

Lemma 1. Suppose that the Bayesian game $\Gamma=\left(T_{i}, X_{i}, u_{i}, p\right)_{i=1}^{N}$ satisfies strong uniform payoff security. If $p$ is absolutely continuous with respect to $p_{1} \otimes \cdots \otimes p_{N}$, then there exists $\mu \in \widehat{D}$ such that the game $G_{\Gamma}^{(\alpha, \mu)}$ defined in (2) is payoff secure for each $\alpha \in[0,1)^{N}$.

Proof. Let $\mu=\left(\mu_{1}, \ldots, \mu_{N}\right) \in \widehat{\mathscr{D}}$ be the measure given by Lemma 5 . Fix $\alpha \in[0,1)^{N}, \varepsilon>0, \sigma \in \mathscr{D}$, and $i$. We must show that there exist $\sigma_{i}^{*} \in \mathscr{D}_{i}$ and a neighborhood $V_{\sigma_{-i}}$ of $\sigma_{-i}$ such that

$$
U_{i}^{(\alpha, \mu)}\left(\sigma_{i}^{*}, \sigma_{-i}^{\prime}\right)>U_{i}^{(\alpha, \mu)}(\sigma)-\varepsilon, \quad \text { for all } \sigma_{-i}^{\prime} \in V_{\sigma_{-i}} .
$$

Define

$$
\widehat{\sigma}=\left(\widehat{\sigma}_{1}, \ldots, \widehat{\sigma}_{N}\right):=\left(\left(1-\alpha_{1}\right) \sigma_{1}+\alpha_{1} \mu_{1}, \ldots,\left(1-\alpha_{N}\right) \sigma_{N}+\alpha_{N} \mu_{N}\right) .
$$

Lemma 5 gives a a sequence $\left(f^{k}\right)$ of $\left(\mathscr{B}\left(X_{i}\right), \mathscr{B}\left(X_{i}\right)\right)$-measurable maps $f^{k}: X_{i} \rightarrow X_{i}$ with $f^{k}\left(X_{i}\right) \in \mathscr{B}\left(X_{i}\right)$ satisfying the following:

(i) For each $\left(t_{i}, x_{i}\right) \in T_{i} \times X_{i}$ and $k$, there is a neighborhood $V_{\widehat{\sigma}_{-i}}$ of $\widehat{\sigma}_{-i}$ such that

$$
\begin{aligned}
\int_{T_{-i}} & \int_{X_{-i}}\left[u_{i}\left(t,\left(f^{k}\left(x_{i}\right), x_{-i}\right)\right) g(t)\right]\left[\underset{j \neq i}{\otimes} \sigma_{j}^{\prime}\left(\cdot \mid t_{j}\right)\right]\left(d x_{-i}\right)\left[\underset{j \neq i}{\otimes} p_{j}\right]\left(d t_{-i}\right) \\
& >\int_{T_{-i}} \int_{X_{-i}}\left[u_{i}(t, x) g(t)\right]\left[\underset{j \neq i}{\otimes} \widehat{\sigma}_{j}\left(\cdot \mid t_{j}\right)\right]\left(d x_{-i}\right)\left[\underset{j \neq i}{\otimes} p_{j}\right]\left(d t_{-i}\right)-\frac{\varepsilon}{4}, \quad \text { for all } \sigma_{-i}^{\prime} \in V_{\widehat{\sigma}_{-i}},
\end{aligned}
$$

where $g$ is a density of $p$ with respect to $p_{1} \otimes \cdots \otimes p_{N}$.

(ii) There exists $K$ such that for each $k \geq K$, there is a neighborhood $V_{\widehat{\sigma}_{-i}}^{\prime}$ of $\widehat{\sigma}_{-i}$ such that

$$
U_{i}\left(\mu_{i}^{k}, \sigma_{-i}^{\prime}\right)<U_{i}\left(\mu_{i}, \sigma_{-i}^{\prime}\right)+\frac{\varepsilon}{2}, \quad \text { for all } \sigma_{-i}^{\prime} \in V_{\widehat{\sigma}_{-i}}^{\prime},
$$

where $\mu_{i}^{k}\left(\cdot \mid t_{i}\right)$ is defined by

$$
\mu_{i}^{k}\left(B \mid t_{i}\right):=\mu_{i}\left(f^{k^{-1}}(B) \mid t_{i}\right)
$$

Define $\widehat{\sigma}_{i}^{k} \in \mathscr{D}_{i}$ and $\sigma_{i}^{k} \in \mathscr{D}_{i}$ via their corresponding regular conditional probability measure as follows:

$$
\widehat{\sigma}_{i}^{k}\left(B \mid t_{i}\right):=\widehat{\sigma}_{i}\left(f^{k^{-1}}(B) \mid t_{i}\right) \quad \text { and } \quad \sigma_{i}^{k}\left(B \mid t_{i}\right):=\sigma_{i}\left(f^{k^{-1}}(B) \mid t_{i}\right) .
$$

Below we show that for each $k$, there exists a neighborhood $V_{\widehat{\sigma}_{-i}}^{\prime \prime}$ of $\widehat{\sigma}_{-i}$ such that for each $\sigma_{-i}^{\prime} \in V_{\widehat{\sigma}_{-i}^{\prime \prime}}^{\prime \prime}$

$$
\int_{T} \int_{X}\left[u_{i}\left(t,\left(f^{k}\left(x_{i}\right), x_{-i}\right)\right) g(t)\right]\left[\widehat{\sigma}_{i}\left(\cdot \mid t_{i}\right) \otimes\left[\underset{j \neq i}{\otimes} \sigma_{j}^{\prime}\left(\cdot \mid t_{j}\right)\right]\right](d x)\left[\underset{j=1}{\otimes} p_{j}\right](d t)>U_{i}(\widehat{\sigma})-\frac{\varepsilon}{2},
$$

implying

$$
U_{i}\left(\widehat{\sigma}^{k}, \sigma_{-i}^{\prime}\right)>U_{i}(\widehat{\sigma})-\frac{\varepsilon}{2}, \quad \text { for all } \sigma_{-i}^{\prime} \in V_{\widehat{\sigma}_{-i}^{\prime}}^{\prime \prime}
$$
that

By (ii), there exists $K$ such that for each $k \geq K$, there is a neighborhood $V_{\widehat{\sigma}_{-i}}^{\prime}$ of $\widehat{\sigma}_{-i}$ such

$$
U_{i}\left(\mu_{i}^{k}, \sigma_{-i}^{\prime}\right)<U_{i}\left(\mu_{i}, \sigma_{-i}^{\prime}\right)+\frac{\varepsilon}{2}, \quad \text { for all } \sigma_{-i}^{\prime} \in V_{\widehat{\sigma}_{-i}}^{\prime} .
$$


Consequently, for $k \geq K$, and for $\sigma_{-i}^{\prime} \in V_{\widehat{\sigma}_{-i}}^{\prime}$,

$$
\begin{aligned}
U_{i}\left(\left(1-\alpha_{i}\right) \sigma_{i}^{k}+\alpha_{i} \mu_{i}, \sigma_{-i}^{\prime}\right) & =\left(1-\alpha_{i}\right) U_{i}\left(\sigma_{i}^{k}, \sigma_{-i}^{\prime}\right)+\alpha_{i} U_{i}\left(\mu_{i}, \sigma_{-i}^{\prime}\right) \\
& >\left(1-\alpha_{i}\right) U_{i}\left(\sigma_{i}^{k}, \sigma_{-i}^{\prime}\right)+\alpha_{i} U_{i}\left(\mu_{i}^{k}, \sigma_{-i}^{\prime}\right)-\frac{\varepsilon}{2} \\
& =U_{i}\left(\widehat{\sigma}_{i}^{k}, \sigma_{-i}^{\prime}\right)-\frac{\varepsilon}{2} .
\end{aligned}
$$

This, together with (16), yields, for $k \geq K$,

$$
U_{i}\left(\left(1-\alpha_{i}\right) \sigma_{i}^{k}+\alpha_{i} \mu_{i}, \sigma_{-i}^{\prime}\right)>U_{i}(\widehat{\sigma})-\varepsilon
$$

for all $\sigma_{-i}^{\prime}$ in some neighborhood of $\widehat{\sigma}_{-i}$. In particular, (14) holds for some neighborhood $V_{\sigma}$ of $\sigma$.

It remains to show that for each $k$, there exists a neighborhood $V_{\widehat{\sigma}_{-i}}^{\prime \prime}$ of $\widehat{\sigma}_{-i}$ such that (15) holds for each $\sigma_{-i}^{\prime} \in V_{\widehat{\sigma}_{-i}}^{\prime \prime}$. For each $k$ and $n \in \mathbb{N}$, define the map $\phi^{(k, n)}: T_{i} \times X_{i} \rightarrow \mathbb{R}$ by

$$
\begin{aligned}
\phi^{(k, n)}\left(t_{i}, x_{i}\right):= & \int_{T_{-i}} \int_{X_{-i}}\left[u_{i}(t, x) g(t)\right]\left[\underset{j \neq i}{\otimes} \widehat{\sigma}_{j}\left(\cdot \mid t_{j}\right)\right]\left(d x_{-i}\right)\left[\underset{j \neq i}{\otimes} p_{j}\right]\left(d t_{-i}\right) \\
& -\inf _{\sigma_{-i}^{\prime} \in N_{\frac{1}{n}}\left(\widehat{\sigma}_{-i}\right)} \int_{T_{-i}} \int_{X_{-i}}\left[u_{i}\left(t,\left(f^{k}\left(x_{i}\right), x_{-i}\right)\right) g(t)\right]\left[\underset{j \neq i}{\otimes} \sigma_{j}^{\prime}\left(\cdot \mid t_{j}\right)\right]\left(d x_{-i}\right)\left[\underset{j \neq i}{\otimes} p_{j}\right]\left(d t_{-i}\right)
\end{aligned}
$$

(here $N_{\frac{1}{n}}\left(\widehat{\sigma}_{-i}\right)$ denotes the $\frac{1}{n}$-neighborhood of $\left.\widehat{\sigma}_{-i}\right)$. Below we show that there exist a $\left(\mathscr{B}\left(T_{i} \times\right.\right.$ $\left.\left.X_{i}\right), \mathscr{B}(\mathbb{R})\right)$-measurable map $\widehat{\phi}^{(k, n)}: T_{i} \times X_{i} \rightarrow \mathbb{R}$ and $\widehat{A} \in \mathscr{B}\left(T_{i} \times X_{i}\right)$ such that

$$
\widehat{\sigma}_{i}(\widehat{A})=0 \quad \text { and } \quad \widehat{\phi}^{(k, n)}\left(t_{i}, x_{i}\right)=\phi^{(k, n)}\left(t_{i}, x_{i}\right) \text { for all }\left(t_{i}, x_{i}\right) \in\left(T_{i} \times X_{i}\right) \backslash \widehat{A} .
$$

Define $\varphi^{k}: T_{i} \times X_{i} \rightarrow \mathbb{R}$ by

$$
\varphi^{k}\left(t_{i}, x_{i}\right):=\lim _{n \rightarrow \infty} \widehat{\phi}^{(k, n)}\left(t_{i}, x_{i}\right)
$$

Observe that by (i) we have

$$
\varphi^{k}\left(t_{i}, x_{i}\right) \leq \frac{\varepsilon}{4}, \quad \text { for each }\left(t_{i}, x_{i}\right) \in\left(T_{i} \times X_{i}\right) \backslash \widehat{A} .
$$

By Egorov's Theorem (see, e.g., Dudley [17, Theorem 7.5.1]), there exists $A \subseteq\left(T_{i} \times X_{i}\right) \backslash \widehat{A}$ such that

$$
\widehat{\sigma}_{i}(A)\left[\sup _{\left(v, v^{\prime}\right) \in \mathscr{D} \times \mathscr{D}}\left(U_{i}(v)-U_{i}\left(v^{\prime}\right)\right)\right]<\frac{\varepsilon}{8}
$$

and $\left(\varphi^{(k, n)}\right)$ converges (as $\left.n \rightarrow \infty\right)$ to $\varphi^{k}$ uniformly on $\left(T_{i} \times X_{i}\right) \backslash(A \cup \widehat{A})$. Consequently, there exists $\bar{n}$ such that for all $n \geq \bar{n}$ and $\left(t_{i}, x_{i}\right) \in\left(T_{i} \times X_{i}\right) \backslash(A \cup \widehat{A}),\left|\varphi^{(k, n)}\left(t_{i}, x_{i}\right)-\varphi^{k}\left(t_{i}, x_{i}\right)\right|<\frac{\varepsilon}{8}$, and so in light of (18) it follows that for all $n \geq \bar{n}, \varphi^{(k, n)}\left(t_{i}, x_{i}\right)<\frac{3 \varepsilon}{8}$ for all $\left(t_{i}, x_{i}\right) \in\left(T_{i} \times X_{i}\right) \backslash(A \cup \widehat{A})$. Hence, there exists a neighborhood $V_{\widehat{\sigma}_{-i}^{\prime \prime}}^{\prime \prime}$ of $\widehat{\sigma}_{-i}$ such that for all $\sigma_{-i}^{\prime} \in V_{\widehat{\sigma}_{-i}}^{\prime \prime}$,

$$
\begin{aligned}
& \int_{T_{-i}} \int_{X_{-i}}\left[u_{i}\left(t,\left(f^{k}\left(x_{i}\right), x_{-i}\right)\right) g(t)\right]\left[\underset{j \neq i}{\otimes} \sigma_{j}^{\prime}\left(\cdot \mid t_{j}\right)\right]\left(d x_{-i}\right)\left[\underset{j \neq i}{\otimes} p_{j}\right]\left(d t_{-i}\right) \\
& >\int_{T_{-i}} \int_{X_{-i}}\left[u_{i}(t, x) g(t)\right]\left[\underset{j \neq i}{\otimes} \widehat{\sigma}_{j}\left(\cdot \mid t_{j}\right)\right]\left(d x_{-i}\right)\left[\underset{j \neq i}{\otimes} p_{j}\right]\left(d t_{-i}\right)-\frac{3 \varepsilon}{8}, \text { for all }\left(t_{i}, x_{i}\right) \in\left(T_{i} \times X_{i}\right) \backslash(A \cup \widehat{A}) .
\end{aligned}
$$

Therefore, using (19), we see that for all $\sigma_{-i}^{\prime} \in V_{\widehat{\sigma}_{-i}}^{\prime \prime},(15)$ holds. 
Finally, we show that we show that there exist a $\left(\mathscr{B}\left(T_{i} \times X_{i}\right), \mathscr{B}(\mathbb{R})\right)$-measurable map $\widehat{\phi}^{(k, n)}$ and $\widehat{A} \in \mathscr{B}\left(T_{i} \times X_{i}\right)$ such that (17) holds. Define $\psi: T_{i} \times X_{i} \rightarrow \mathbb{R}$ and $\vartheta^{(k, n)}: T_{i} \times X_{i} \rightarrow \mathbb{R}$ by

$$
\psi\left(t_{i}, x_{i}\right):=\int_{T_{-i}} \int_{X_{-i}}\left[u_{i}(t, x) g(t)\right]\left[\underset{j \neq i}{\otimes} \widehat{\sigma}_{j}\left(\cdot \mid t_{j}\right)\right]\left(d x_{-i}\right)\left[\underset{j \neq i}{\otimes} p_{j}\right]\left(d t_{-i}\right)
$$

and

$$
\vartheta^{(k, n)}\left(t_{i}, x_{i}\right):=\inf _{\sigma_{-i}^{\prime} \in N_{\frac{1}{n}}\left(\widehat{\sigma}_{-i}\right)} \int_{T_{-i}} \int_{X_{-i}}\left[u_{i}\left(t,\left(f^{k}\left(x_{i}\right), x_{-i}\right)\right) g(t)\right]\left[\underset{j \neq i}{\otimes} \sigma_{j}^{\prime}\left(\cdot \mid t_{j}\right)\right]\left(d x_{-i}\right)\left[\underset{j \neq i}{\otimes} p_{j}\right]\left(d t_{-i}\right),
$$

so that $\phi^{(k, n)}=\psi^{(k, n)}-\vartheta^{(k, n)}$. It suffices to show that $\psi$ is $\left(\mathscr{B}\left(T_{i} \times X_{i}\right), \mathscr{B}(\mathbb{R})\right)$-measurable and that there exist a $\left(\mathscr{B}\left(T_{i} \times X_{i}\right), \mathscr{B}(\mathbb{R})\right)$-measurable map $\widehat{\vartheta}^{(k, n)}: T_{i} \times X_{i} \rightarrow \mathbb{R}$ and $\widehat{A} \in \mathscr{B}\left(T_{i} \times X_{i}\right)$ such that $\widehat{\sigma}_{i}(\widehat{A})=0$ and $\widehat{\vartheta}^{(k, n)}\left(t_{i}, x_{i}\right)=\vartheta^{(k, n)}\left(t_{i}, x_{i}\right)$ for all $\left(t_{i}, x_{i}\right) \in\left(T_{i} \times X_{i}\right) \backslash \widehat{A}$.

To see that $\psi$ is $\left(\mathscr{B}\left(T_{i} \times X_{i}\right), \mathscr{B}(\mathbb{R})\right)$-measurable, define $\psi: \Delta(T \times X) \rightarrow \mathbb{R}$ by

$$
\boldsymbol{\psi}(\varrho):=\int_{T \times X}\left[u_{i}(t, x) g(t)\right] \varrho(d(t, x)) .
$$

Since $u_{i}$ is $(\mathscr{B}(T \times X), \mathscr{B}(\mathbb{R}))$-measurable, the map $\boldsymbol{\psi}$ is $(\mathscr{B}(\Delta(T \times X)), \mathscr{B}(\mathbb{R}))$-measurable (see, e.g., Aliprantis and Border [1, Theorem 15.13]). Let $\Delta^{p}(T \times X)$ be the set of all product measures in $\Delta(T \times X)$ (i.e., $v \in \Delta^{p}(T \times X)$ if and only if $v=v_{1} \otimes \cdots \otimes v_{N}$ for some $\left(v_{1}, \ldots, v_{N}\right) \in$ $\left.\times_{j} \Delta\left(T_{j} \times X_{j}\right)\right)$. Since $\Delta^{p}(T \times X)$ is closed in $\Delta(T \times X)$, the map $\left.\boldsymbol{\psi}\right|_{\Delta^{p}(T \times X)}$ is $\left(\mathscr{B}\left(\Delta^{p}(T \times\right.\right.$ $X)), \mathscr{B}(\mathbb{R}))$-measurable. ${ }^{2}$ Hence, because the map $\left(v_{1}, \ldots, v_{N}\right) \in \times_{j} \Delta\left(T_{j} \times X_{j}\right) \mapsto v_{1} \otimes \cdots \otimes v_{N} \in$ $\Delta^{p}(T \times X)$ is continuous, it follows that the map

$$
\left(v_{1}, \ldots, v_{N}\right) \in \times_{j} \Delta\left(T_{j} \times X_{j}\right) \mapsto \boldsymbol{\psi}\left(v_{1} \otimes \cdots \otimes v_{N}\right)
$$

is $\left(\mathscr{B}\left(\times_{j} \Delta\left(T_{j} \times X_{j}\right)\right), \mathscr{B}(\mathbb{R})\right)$-measurable, and hence $\left(\mathscr{B}\left(\Delta\left(T_{i} \times X_{i}\right)\right) \otimes \mathscr{B}\left(\times_{j \neq i} \Delta\left(T_{j} \times X_{j}\right)\right), \mathscr{B}(\mathbb{R})\right)$ measurable (see, e.g., Aliprantis and Border [1, Theorem 4.44]). ${ }^{3}$ Therefore, the map $v_{i} \in$ $\Delta\left(T_{i} \times X_{i}\right) \mapsto \boldsymbol{\psi}\left(v_{i} \otimes\left[\otimes_{j \neq i} \widehat{\sigma}_{j}\right]\right)$ is $\left(\mathscr{B}\left(\Delta\left(T_{i} \times X_{i}\right)\right), \mathscr{B}(\mathbb{R})\right)$-measurable (see, e.g., Aliprantis and Border [1, Theorem 4.48]). Now let $\delta_{\left(t_{i}, x_{i}\right)}$ denote the Dirac measure in $\Delta\left(T_{i} \times X_{i}\right)$ with support $\left\{\left(t_{i}, x_{i}\right)\right\}$. The set $\left\{\delta_{\left(t_{i}, x_{i}\right)}:\left(t_{i}, x_{i}\right) \in T_{i} \times X_{i}\right\}$ is closed in $\Delta\left(T_{i} \times X_{i}\right)$ (see, e.g., Aliprantis and Border [1, Theorem 15.8]), and so the map $v_{i} \in\left\{\delta_{\left(t_{i}, x_{i}\right)}:\left(t_{i}, x_{i}\right) \in T_{i} \times X_{i}\right\} \mapsto \psi\left(v_{i} \otimes\left[\otimes_{j \neq i} \widehat{\sigma}_{j}\right]\right)$ is $\left(\mathscr{B}\left(\left\{\delta_{\left(t_{i}, x_{i}\right)}:\left(t_{i}, x_{i}\right) \in T_{i} \times X_{i}\right\}\right), \mathscr{B}(\mathbb{R})\right)$-measurable. Because the map $\left(t_{i}, x_{i}\right) \in T_{i} \times X_{i} \mapsto$ $\delta_{\left(t_{i}, x_{i}\right)}$ is an embedding ( $c f$. Aliprantis and Border [1, Theorem 15.8]), it follows that $\psi$ is $\left(\mathscr{B}\left(T_{i} \times X_{i}\right), \mathscr{B}(\mathbb{R})\right)$-measurable.

To see that there exist a $\left(\mathscr{B}\left(T_{i} \times X_{i}\right), \mathscr{B}(\mathbb{R})\right)$-measurable map $\widehat{\vartheta}^{(k, n)}: T_{i} \times X_{i} \rightarrow \mathbb{R}$ and $\widehat{A} \in \mathscr{B}\left(T_{i} \times X_{i}\right)$ such that

$$
\widehat{\sigma}_{i}(\widehat{A})=0 \quad \text { and } \quad \widehat{\vartheta}^{(k, n)}\left(t_{i}, x_{i}\right)=\vartheta^{(k, n)}\left(t_{i}, x_{i}\right) \text { for all }\left(t_{i}, x_{i}\right) \in\left(T_{i} \times X_{i}\right) \backslash \widehat{A},
$$

define $\boldsymbol{\vartheta}^{k}: \Delta(T \times X) \rightarrow \mathbb{R}$ by

$$
\boldsymbol{\vartheta}^{k}(\varrho):=\int_{T \times X}\left[u_{i}\left(t,\left(f^{k}\left(x_{i}\right), x_{-i}\right)\right) g(t)\right] \varrho(d(t, x)) .
$$

\footnotetext{
${ }^{2}$ To see that $\Delta^{p}(T \times X)$ is closed in $\Delta(T \times X)$, let $\left(v^{n}=v_{1}^{n} \otimes \cdots \otimes v_{N}^{n}\right)$ be a sequence in $\Delta^{p}(T \times X)$ with $v^{n} \underset{w}{\rightarrow} v \in \Delta(T \times X)$. Then $v^{n}\left(A_{1} \times \cdots \times A_{N}\right) \rightarrow v\left(A_{1} \times \cdots \times A_{N}\right)$, where for each $j, A_{j}$ is any $v_{j}$-continuity subset of $\stackrel{w}{T_{j}} \times X_{j}$ and $v_{j}$ denotes the marginal projection of $v$ into $T_{j} \times X_{j}$ (see, e.g., Billingsley [7, Theorem 2.8(i)]). In particular, letting $v_{j}^{n}$ represent the marginal projection of $v^{n}$ into the factor $T_{j} \times X_{j}, v_{j}^{n}\left(A_{j}\right) \rightarrow v_{j}\left(A_{j}\right)$ for every $v_{j}$-continuity set $A_{j}$, and so it follows from the Portmanteau Theorem (e.g., see Billingsley [7, Theorem 2.1]) that $v_{j}^{n} \underset{w}{\longrightarrow} v_{j}$ for each $j$. Applying Theorem 2.8(ii) in Billingsley [7] we see that $v=v_{1} \otimes \cdots \otimes v_{N}$.

${ }^{3}$ The continuity of the map $\left(v_{1}, \ldots, v_{N}\right) \in \times_{j} \Delta\left(T_{j} \times X_{j}\right) \mapsto v_{1} \otimes \cdots \otimes v_{N} \in \Delta^{p}(T \times X)$ follows from Theorem 2.8(ii) in Billingsley [7].
} 
Reasoning as in the preceding paragraph one can show that the map

$$
\left(\left(t_{i}, x_{i}\right), v_{-i}\right) \in\left(T_{i} \times X_{i}\right) \times\left[\underset{j \neq i}{\times} \Delta\left(T_{j} \times X_{j}\right)\right] \mapsto \boldsymbol{\vartheta}^{k}\left(\delta_{\left(t_{i}, x_{i}\right)} \otimes\left[\underset{j \neq i}{\otimes} v_{j}\right]\right)
$$

is $\left(\mathscr{B}\left(T_{i} \times X_{i}\right) \otimes \mathscr{B}\left(\times_{j \neq i} \Delta\left(T_{j} \times X_{j}\right)\right), \mathscr{B}(\mathbb{R})\right)$-measurable. Let $\mathscr{B}^{\widehat{\sigma}_{i}}\left(T_{i} \times X_{i}\right)$ be the $\widehat{\sigma}_{i}$-completion of $\mathscr{B}\left(T_{i} \times X_{i}\right)$. Then the map in (21) is $\left(\mathscr{B}^{\widehat{\sigma}_{i}}\left(T_{i} \times X_{i}\right) \otimes \mathscr{B}\left(\times_{j \neq i} \Delta\left(T_{j} \times X_{j}\right)\right), \mathscr{B}(\mathbb{R})\right)$-measurable, and since $\mathscr{B}^{\widehat{\sigma}_{i}}\left(T_{i} \times X_{i}\right)$ equals its universal completion, it follows from the proof of the Theorem in Carbonell-Nicolau [11] that the map

$$
\left(\left(t_{i}, x_{i}\right), v_{-i}\right) \in\left(T_{i} \times X_{i}\right) \times\left[\underset{j \neq i}{\times} \Delta\left(T_{j} \times X_{j}\right)\right] \mapsto \inf _{v_{-i}^{\prime} \in N_{\frac{1}{n}}\left(v_{-i}\right)} \boldsymbol{\vartheta}^{k}\left(\delta_{\left(t_{i}, x_{i}\right)} \otimes\left[\underset{j \neq i}{\otimes} v_{j}^{\prime}\right]\right)
$$

is $\left(\mathscr{B}^{\widehat{\sigma}_{i}}\left(T_{i} \times X_{i}\right) \otimes \mathscr{B}\left(\times_{j \neq i} \Delta\left(T_{j} \times X_{j}\right)\right), \mathscr{B}(\mathbb{R})\right)$-measurable (here $N_{\frac{1}{n}}\left(v_{-i}\right)$ denotes the $\frac{1}{n}$-neighborhood of $v_{-i}$ in $\mathscr{D}_{-i}$ ), and consequently the map

$$
\left(t_{i}, x_{i}\right) \in\left(T_{i} \times X_{i}\right) \mapsto \inf _{v_{-i}^{\prime} \in N_{\frac{1}{n}}\left(\widehat{\sigma}_{-i}\right)} \boldsymbol{\vartheta}^{k}\left(\delta_{\left(t_{i}, x_{i}\right)} \otimes\left[\underset{j \neq i}{\otimes} v_{j}^{\prime}\right]\right)
$$

is $\left(\mathscr{B}^{\widehat{\sigma}_{i}}\left(T_{i} \times X_{i}\right), \mathscr{B}(\mathbb{R})\right)$-measurable (cf. Aliprantis and Border [1, Theorem 4.48]). Now Theorem 10.35 in Aliprantis and Border [1] gives a $\left(\mathscr{B}\left(T_{i} \times X_{i}\right), \mathscr{B}(\mathbb{R})\right)$-measurable map $\widehat{\vartheta}^{(k, n)}: T_{i} \times X_{i} \rightarrow \mathbb{R}$ and $\widehat{A} \in \mathscr{B}\left(T_{i} \times X_{i}\right)$ satisfying (20).

\section{A.2 Proof of Lemma 2}

We restate Lemma 2 here for the convenience of the reader.

Lemma 2. Given a Bayesian game $\left(T_{i}, X_{i}, u_{i}, p\right)_{i=1}^{N}$, suppose that for each $t \in T$, the map $\sum_{i=1}^{N} u_{i}(t, \cdot): X \rightarrow \mathbb{R}$ is upper semicontinuous. Suppose further that $p$ is absolutely continuous with respect to $p_{1} \otimes \cdots \otimes p_{N}$. Then the map $\sum_{i=1}^{N} U_{i}(\cdot): \mathscr{D} \rightarrow \mathbb{R}$ is upper semicontinuous.

Proof. Fix $\sigma \in \mathscr{D}$ and $\epsilon>0$. Let $f$ be a density of $p$ with respect to $P:=p_{1} \otimes \cdots \otimes p_{N}$. We must show that there exists a neighborhood $V_{\sigma}$ of $\sigma$ such that

$$
\sum_{i=1}^{N} U_{i}\left(\sigma^{\prime}\right)<\sum_{i=1}^{N} U_{i}(\sigma)+\epsilon, \text { for all } \sigma^{\prime} \in V_{\sigma}
$$

Let $\mathscr{P}^{*}$ be the set of all $\mu$ in $\Delta(T \times X)$ that take the form

$$
\mu(A \times B)=\int_{A} \mu(B \mid t) P(d t)
$$

for all $A \times B \subseteq T \times X$ in $\mathscr{B}(T \times X)$ and for some $\mu: T \rightarrow \Delta(X)$. Endow $\mathscr{P}^{*}$ with the weak-strong topology (cf. Balder [5]).

The map $\mu \in \mathscr{D} \mapsto P \otimes \mu \in \mathscr{P}^{*}$, where $P \otimes \mu$ is defined by

$$
[P \otimes \mu](A \times B):=\int_{A}\left[\mu_{1}\left(\cdot \mid t_{1}\right) \otimes \cdots \otimes \mu_{N}\left(\cdot \mid t_{N}\right)\right](B) P(d t)
$$

is continuous. Indeed, if $\left(\mu^{n}\right)$ is a sequence in $\mathscr{D}$ with limit point $\mu \in \mathscr{D}$, then by Theorem 2.8(ii) of Billingsley [7] we have $\mu_{1}^{n} \otimes \cdots \otimes \mu_{N}^{n} \vec{w} \mu_{1} \otimes \cdots \otimes \mu_{N}$. In addition, since for $A=A_{1} \times \cdots \times A_{N}$ and $B=B_{1} \times \cdots \times B_{N}$ we have

$$
\begin{aligned}
{\left[P \otimes \mu^{n}\right](A \times B) } & =\int_{A}\left[\mu_{1}^{n}\left(\cdot \mid t_{1}\right) \otimes \cdots \otimes \mu_{N}^{n}\left(\cdot \mid t_{N}\right)\right](B) P(d t) \\
& =\int_{A_{N}} \cdots \int_{A_{1}} \mu_{1}^{n}\left(B_{1} \mid t_{1}\right) p_{1}\left(d t_{1}\right) \cdots \mu_{N}^{n}\left(B_{N} \mid t_{N}\right) p_{N}\left(d t_{N}\right) \\
& =\left[\mu_{1}^{n} \otimes \cdots \otimes \mu_{N}^{n}\right](A \times B),
\end{aligned}
$$


it follows that $P \otimes \mu^{n}=\mu_{1}^{n} \otimes \cdots \otimes \mu_{N}^{n}$. Similarly, we have $P \otimes \mu=\mu_{1} \otimes \cdots \otimes \mu_{N}$. Consequently, $P \otimes \mu^{n} \underset{w}{\rightarrow} P \otimes \mu$, and this, together with the fact that $\left[P \otimes \mu^{n}\right](\cdot \times B)=[P \otimes \mu](\cdot \times B)$ for each $B \in \mathscr{B}(X)$ and $n$ implies (by Theorem 3.7(viii) of Schäl [25]) that $P \otimes \mu^{n}$ converges to $P \otimes \mu$ with respect to the weak-strong topology.

By Theorem 3.1 in Balder [5], there exists a neighborhood $V_{\sigma}^{\prime}$ of $\sigma$ in $\mathscr{P}^{*}$ (with respect to the weak-strong topology) such that

$$
\int_{T \times X}\left[\sum_{i=1}^{N}\left[u_{i}(t, x) f(t)\right]\right] v(d(t, x))<\int_{T \times X}\left[\sum_{i=1}^{N}\left[u_{i}(t, x) f(t)\right]\right][P \otimes \sigma](d(t, x))+\epsilon \text {, for all } v \in V_{\sigma}^{\prime} .
$$

Therefore, since the map $\mu \in \mathscr{D} \mapsto P \otimes \mu \in \mathscr{P}^{*}$ is continuous, it follows that there is a neighbor$\operatorname{hood} V_{\sigma}$ of $\sigma$ in $\mathscr{D}$ such that

$$
\int_{T \times X}\left[\sum_{i=1}^{N}\left[u_{i}(t, x) f(t)\right]\right]\left[P \otimes \sigma^{\prime}\right](d(t, x))<\int_{T \times X}\left[\sum_{i=1}^{N}\left[u_{i}(t, x) f(t)\right]\right][P \otimes \sigma](d(t, x))+\epsilon \text {, for all } \sigma^{\prime} \in V_{\sigma} .
$$

This implies (22).

\section{A.3 Proof of Lemma 3}

Lemma 3 is restated here for the convenience of the reader.

Lemma 3. Suppose that $\Gamma=\left(T_{i}, X_{i}, u_{i}, p\right)_{i=1}^{N}$ is a Bayesian game satisfying generic entire payoff security. Then $\Gamma$ satisfies strong uniform payoff security.

Proof. Let $\mu=\left(\mu_{1}, \ldots, \mu_{N}\right) \in \widetilde{\mathscr{D}}$ be the profile of measures given by the generic local equi-upper semicontinuity condition. Fix $\varepsilon>0$ and $i$. By the generic entire payoff security condition, for each $x_{i} \in X_{i}$ and $k \in \mathbb{N}$, there exist $h^{k}\left(x_{i}\right) \in X_{i}$ and $\gamma^{k}\left(x_{i}\right)>0$ such that for every $(t, z) \in T \times X$, there is a neighborhood $V_{z_{-i}}$ of $z_{-i}$ such that

$$
\begin{array}{rr}
u_{i}\left(t,\left(h^{k}\left(x_{i}\right), y_{-i}\right)\right)>u_{i}(t, z)-\varepsilon & \text { for all } y_{-i} \in V_{z_{-i}}, \\
& \text { if } x_{i} \in K_{i} \cup C_{i}, \\
h^{k}\left(x_{i}\right) \in N_{\frac{1}{k}}\left(x_{i}\right) \text { and } u_{i}\left(t,\left(h^{k}\left(x_{i}\right), y_{-i}\right)\right)>u_{i}\left(t,\left(z_{i}^{\prime}, z_{-i}\right)\right)-\varepsilon & \text { for all }\left(z_{i}^{\prime}, y_{-i}\right) \in N_{\gamma^{k}\left(x_{i}\right)}\left(x_{i}\right) \times V_{z_{-i}}, \\
& \text { if } x_{i} \in A_{i} \backslash C_{i},
\end{array}
$$

where $C_{i}$ is a countable subset of $A_{i}$. In addition, there is no loss of generality in assuming that $\gamma^{k}\left(x_{i}\right)<\frac{1}{k}$ and, since $\Gamma$ is entirely payoff secure over $\times_{j=1}^{N} K_{j}$, one may take $h^{k}\left(x_{i}\right)=x_{i}$ for $x_{i} \in K_{i}$.

Now since $A_{i} \backslash C_{i} \subseteq X_{i}$ and $X_{i}$ is compact and metric, $A_{i} \backslash C_{i}$ is separable, and so there is a countable subset $\left\{x_{i}^{(k, 1)}, x_{i}^{(k, 2)}, \ldots\right\}$ of $A_{i} \backslash C_{i}$ such that

$$
\bigcup_{l=1}^{\infty}\left(N_{\gamma^{k}\left(x_{i}^{(k, l)}\right)}\left(x_{i}^{(k, l)}\right) \cap\left(A_{i} \backslash C_{i}\right)\right)=\bigcup_{x_{i} \in A_{i} \backslash C_{i}}\left(N_{\gamma^{k}\left(x_{i}\right)}\left(x_{i}\right) \cap\left(A_{i} \backslash C_{i}\right)\right) .
$$

Define $V^{(k, 1)}, V^{(k, 2)}, \ldots$ recursively as follows:

$$
V^{(k, 1)}:=N_{\gamma^{k}\left(x_{i}^{(k, 1)}\right)}\left(x_{i}^{(k, 1)}\right) \cap\left(A_{i} \backslash C_{i}\right)
$$

and

$$
V^{(k, l)}:=\left(N_{\gamma^{k}\left(x_{i}^{(k, l)}\right)}\left(x_{i}^{(k, l)}\right) \cap\left(A_{i} \backslash C_{i}\right)\right) \backslash\left(\bigcup_{m=1}^{l-1} V^{(k, m)}\right), \quad l \in\{2,3, \ldots\} .
$$


Now define, for each $k, f^{k}: X_{i} \rightarrow X_{i}$ by

$$
f^{k}\left(x_{i}\right):= \begin{cases}h^{k}\left(x_{i}^{(k, l)}\right) & \text { if } x_{i} \in V^{(k, l)} \\ h^{k}\left(x_{i}\right) & \text { if } x_{i} \in C_{i} \cup K_{i} .\end{cases}
$$

Observe that

$$
f^{k}\left(X_{i}\right)=f^{k}\left(A_{i} \backslash C_{i}\right) \cup f^{k}\left(C_{i} \cup K_{i}\right)=\left\{h^{k}\left(x^{(k, 1)}\right), h^{k}\left(x^{(k, 2)}\right), \ldots\right\} \cup f^{k}\left(C_{i} \cup K_{i}\right),
$$

and so $f^{k}\left(X_{i}\right)$ is countable. Therefore, given $B \in \mathscr{B}\left(X_{i}\right), B \cap f^{k}\left(X_{i}\right)$ is countable, and it can be expressed as a disjoint union $B^{\prime} \cup B^{\prime \prime}$, where $B^{\prime} \subseteq A_{i} \backslash C_{i}$ and $B^{\prime \prime} \subseteq C_{i} \cup K_{i}$. Since

$$
f^{k^{-1}}(B)=f^{k^{-1}}\left(B^{\prime}\right) \cup f^{k^{-1}}\left(B^{\prime \prime}\right)=\left(\bigcup_{l=1}^{\infty} V^{(k, l)}\right) \cup f^{k^{-1}}\left(B^{\prime \prime}\right)
$$

and $f^{k^{-1}}\left(B^{\prime \prime}\right)$ is countable, it follows that $f^{k^{-1}}(B) \in \mathscr{B}\left(X_{i}\right)$, and so $f^{k}$ is $\left(\mathscr{B}\left(X_{i}\right), \mathscr{B}\left(X_{i}\right)\right)$ measurable.

To see that item (a) of Definition 10 holds, fix $k$ and $(t, x) \in T \times X$. If $x_{i} \in C_{i} \cup K_{i}$, it is clear that there exists a neighborhood $V_{x_{-i}}$ of $x_{-i}$ such that

$$
u_{i}\left(t,\left(f^{k}\left(x_{i}\right), y_{-i}\right)\right)>u_{i}(t, x)-\varepsilon, \quad \text { for all } y_{-i} \in V_{x_{-i}} .
$$

Now suppose that $x_{i} \in A_{i} \backslash C_{i}$. Then $x_{i} \in V^{(k, l)}$ for some $l$ and $f^{k}\left(x_{i}\right)=h^{k}\left(x_{i}^{(k, l)}\right)$. Therefore, since there is a neighborhood $V_{x_{-i}}$ of $x_{-i}$ such that

$$
u_{i}\left(t,\left(h^{k}\left(x_{i}^{(k, l)}\right), y_{-i}\right)\right)>u_{i}\left(t,\left(x_{i}^{\prime}, x_{-i}\right)\right)-\varepsilon, \quad \text { for all }\left(x_{i}^{\prime}, y_{-i}\right) \in N_{\gamma^{k}\left(x_{i}^{(k, l)}\right)}\left(x_{i}\right) \times V_{x_{-i}},
$$

and because $x_{i} \in N_{\gamma^{k}\left(x_{i}^{(k, l)}\right)}\left(x_{i}\right)$, one obtains (23).

To see that item (b) of Definition 10 holds, fix $\left(t, x_{-i}\right) \in T \times X_{-i}$ and let $Y_{i}$ be the set given by the generic local equi-upper semicontinuity condition. Set $Y_{i}^{\prime}:=Y_{i} \backslash C_{i}$. Then $\mu_{i}\left(Y_{i}^{\prime} \mid t_{i}\right)=1$. In addition, given $x_{i} \in Y_{i}^{\prime}, f^{k}\left(x_{i}\right)=x_{i}$ if $x_{i} \in K_{i}$ and $f^{k}\left(x_{i}\right)=h^{k}\left(x_{i}^{(k, l)}\right), h^{k}\left(x_{i}^{(k, l)}\right) \in N_{\frac{1}{k}}\left(x_{i}^{(k, l)}\right)$, and $x_{i} \in V^{(k, l)} \subseteq N_{\gamma^{k}\left(x_{i}^{(k, l)}\right)}\left(x_{i}^{(k, l)}\right) \subseteq N_{\frac{1}{k}}\left(x_{i}^{(k, l)}\right)$ if $x_{i} \in A_{i} \backslash C_{i}$. Consequently, $f^{k}\left(x_{i}\right) \rightarrow x_{i}$ for every $x_{i} \in Y_{i}^{\prime}$. Now given $x_{i} \in Y_{i}^{\prime}$, the generic local equi-upper semicontinuity condition gives a neighborhood $V_{x_{i}}$ of $x_{i}$ such that for every $y_{i} \in V_{x_{i}}$, there is a neighborhood $V_{x_{-i}}^{\prime}$ of $x_{-i}$ such that

$$
u_{i}\left(t,\left(y_{i}, y_{-i}\right)\right)<u_{i}\left(t,\left(x_{i}, y_{-i}\right)\right)+\varepsilon, \quad \text { for all } y_{-i} \in V_{x_{-i}}^{\prime} .
$$

Since $f^{k}\left(x_{i}\right) \rightarrow x_{i}$, there exists $K$ such that for all $k \geq K$, there is a neighborhood $V_{x_{-i}}^{\prime}$ of $x_{-i}$ such that

$$
u_{i}\left(t,\left(f^{k}\left(x_{i}\right), y_{-i}\right)\right)<u_{i}\left(t,\left(x_{i}, y_{-i}\right)\right)+\varepsilon, \quad \text { for all } y_{-i} \in V_{x_{-i}}^{\prime}
$$

as desired.

\section{References}

[1] Aliprantis, C.D., and K.C. Border (2006), Infinite Dimensional Analysis. Berlin: SpringerVerlag.

[2] Al-Najjar, N. (1995), "Strategically stable equilibria in games with infinitely many pure strategies," Mathematical Social Sciences 29, 151-164. 
[3] Averna, D. (1994), "Lusin type theorems for multifunctions, Scorza Dragoni's property and Carathéodory selections," Bollettino dell'Unione Matematica Italiana 8-A, 193-202.

[4] Bajoori, E., Flesch, J., and D. Vermeulen (2013), "Perfect equilibrium in games with compact action spaces," Games and Economic Behavior 82, 490-502.

[5] Balder, E.J. (2001), "On ws-convergence of product measures," Mathematics of Operations Research 26, 494-518.

[6] Bertsekas, D.P., and S.E. Shreve (1996), Stochastic Optimal Control: The Discrete-Time Case. Belmont, Massachusetts: Athena Scientific.

[7] Billingsley, P. (1999), Convergence of Probability Measures. New York: John Wiley \& Sons.

[8] Carbonell-Nicolau, O. (2011), "On the existence of pure-strategy perfect equilibrium in discontinuous games," Games and Economic Behavior 71, 23-48.

[9] Carbonell-Nicolau, O. (2011), "The existence of perfect equilibrium in discontinuous games," Games 2, 235-256.

[10] Carbonell-Nicolau, O. (2011), "Perfect and limit admissible perfect equilibria in discontinuous games," Journal of Mathematical Economics 47, 531-540.

[11] Carbonell-Nicolau, O. (2014), "Semicontinuous integrands as jointly measurable maps," Commentationes Mathematicae Universitatis Carolinae 55, 189-193.

[12] Carbonell-Nicolau, O. (2014), "On essential, (strictly) perfect equilibria," Journal of Mathematical Economics 54, 157-162.

[13] Carbonell-Nicolau, O., and R.P. McLean (2013), "Approximation results for discontinuous games with an application to equilibrium refinement," Economic Theory 54, 1-26.

[14] Carbonell-Nicolau, O., and R.P. McLean (2014), "Refinements of Nash equilibrium in potential games," Theoretical Economics 9, 555-582.

[15] Carbonell-Nicolau, O., and R.P. McLean (2015), "On equilibrium refinements in supermodular games," International Journal of Game Theory 44, 869-890.

[16] Carbonell-Nicolau, O., and R.P. McLean (2017), "On the existence of Nash equilibrium in Bayesian games," Mathematics of Operations Research, forthcoming.

[17] Dudley, R.M. (2004), Real Analysis and Probability. Cambridge, MA: Cambridge University Press.

[18] Krishna, V., and J. Morgan (1997), "An analysis of the war of attrition and the all-pay auction," Journal of Economic Theory 72, 343-362.

[19] Milgrom, P.R., and R.J. Weber (1985), "Distributional strategies for games with incomplete information," Mathematics of Operations Research 10, 619-632.

[20] Parthasarathy, K.R., Ranga Rao, R., and S.R.S. Varadhan (1962), "On the category of indecomposable distributions on topological groups," Transactions of the American Mathematical Society 102, 200-217. 
[21] Prokhorov, Y.V. (1956), "Convergence of random processes and limit theorems in probability theory," Theory of Probability and its Applications 1, 157-214.

[22] Reny, P.J. (1999), "On the existence of pure and mixed strategy Nash equilibria in discontinuous games," Econometrica 67, 1029-1056.

[23] Selten, R. (1975), "Reexamination of the perfectness concept for equilibrium points in extensive games," International Journal of Game Theory 4, 25-55.

[24] Simon, L.K., and M.B. Stinchcombe (1995), "Equilibrium refinement for infinite normalform games," Econometrica 63, 1421-1443.

[25] Schäl, M. (1975), "On dynamic programming: compactness of the space of policies," Stochastic Processes and their Applications 3, 345-364.

[26] van Damme, E.E.C. (2002), Stability and Perfection of Nash Equilibria. Berlin: SpringerVerlag. 